\title{
Angela Scalabrin
}

Justiça organizacional e desfechos negativos em saúde:

uma revisão sistemática

Dissertação apresentada à Faculdade de Medicina da Universidade de São Paulo para obtenção do título de Mestre em Ciências

Programa de Saúde Coletiva

Orientador: Prof. Dr. Paulo Rossi Menezes

\section{São Paulo}


Dados Internacionais de Catalogação na Publicação (CIP)

Preparada pela Biblioteca da

Faculdade de Medicina da Universidade de São Paulo

(C)reprodução autorizada pelo autor

Scalabrin, Angela

Justiça organizacional e desfechos negativos em

saúde : uma revisão sistemática / Angela Scalabrin.

- Sáo Paulo, 2021.

Universidade de São Paulo.

Programa de Saúde Coletiva.

Orientador: Paulo Rossi Menezes.

Descritores: 1.Justiça organizacional 2.Saúde do trabalhador 3.Doenças cardiovasculares 4. Doença das coronárias 5. Transtornos cerebrovasculares

6. Hipertensão 7.Doenças do sistema endócrino

USP/FM/DBD-268/21

Responsável: Erinalva da Conceição Batista, CRB-8 6755 


\section{AGRADECIMENTOS}

O mestrado foi um período de vivência muito intensa, intercalando momentos de alegria e otimismo, com momentos de fragilidade e inquietação. Não bastassem as vicissitudes normais deste período, adversidades políticas e sanitárias deixaram esse momento ainda mais turbulento. Nesse contexto, gostaria de agradecer a todas as pessoas que contribuíram para a minha trajetória. Deixo um agradecimento especial a estes que de uma maneira mais significativa participaram deste processo.

Aos meus pais, Olidair e Maristela, pelo amor incondicional e incentivo constante.

À minha irmã, Cristina, pela amizade, estímulo e parceria.

À minha esposa, Andréa, por todo o apoio e amor durante esta trajetória. Você torna meu mundo mais feliz.

Ao meu orientador, Prof. Dr. Paulo Rossi Menezes, pela oportunidade e por ter acreditado no meu potencial.

À Universidade de São Paulo, instituição pública, gratuita e de qualidade, pelo seu papel na educação e a ciência deste país. 


\section{NORMALIZAÇÃO ADOTADA}

Esta dissertação está de acordo com as seguintes normas, em vigor no momento desta publicação:

Universidade de São Paulo. Sistema Integrado de Bibliotecas da USP. Diretrizes para apresentação de dissertações e teses da USP: parte IV (Vancouver)/ Sistema Integrado de Bibliotecas da USP; Vânia Martins Bueno de Oliveira Funaro [et al.]. 3.ed. rev. ampl. mod. São Paulo: SIBi/USP, 2016.

Referências: adaptado de International Committee of Medical Journals Editors (Vancouver).

Abreviaturas dos títulos dos periódicos de acordo com List of Journals Indexed in Index Medicus. 


\section{RESUMO}

Scalabrin A. Justiça organizacional e desfechos negativos em saúde: uma revisão sistemática [dissertação]. São Paulo: Faculdade de Medicina, Universidade de São Paulo; 2021.

Introdução: O estresse no trabalho tem repercussões para a saúde física e/ou mental dos trabalhadores. Entre os modelos conceituais de estresse no trabalho, o de Justiça Organizacional é um dos que têm sido investigados atualmente. Esse modelo permite identificar aspectos do contexto de trabalho relacionados à percepção de justiça nas dimensões distributiva (distribuição de recursos), procedural (tomada de decisão) e interacional (qualidade da comunicação e tratamento interpessoal). Vários estudos investigaram associações da percepção de justiça organizacional e desfechos em saúde, em particular, seus impactos sobre a saúde mental do trabalhador. Alguns estudos investigaram as repercussões de vivenciar injustiça organizacional sobre o aparelho cardiovascular e metabolismo dos trabalhadores. Entretanto, a sistematização da informação científica disponível sobre esse tema ainda é escassa. Objetivo: Revisar sistematicamente evidências de associação entre percepção de justiça organizacional e doença cardiovascular e metabólica em trabalhadores adultos. Métodos: As bases de dados investigadas foram MEDLINE, EMBASE, LILACS. A estratégia de busca utilizou cinco combinações para cada base, que incluíam a exposição (Justiça Organizacional) e um desfecho (doença arterial coronariana; doença cerebrovascular; hipertensão arterial sistêmica; diabetes mellitus; doença cardiovascular). Os critérios de elegibilidade incluíam publicações originais a partir de estudos observacionais (coorte, transversal ou caso-controle), publicados em Inglês, Português ou Espanhol. Os títulos selecionados foram avaliados por dois revisores, em caso de dissenso um terceiro revisor foi chamado. A qualidade dos estudos foi avaliada por meio dos critérios do Study Quality Assessment Tools do National Institute of Health. Resultados: Foram identificados 1.932 títulos nas bases de dados investigados. Restaram 19 artigos para avaliação na íntegra, dos quais 12 avaliaram estresse no trabalho utilizando outros modelos que não o de justiça organizacional e um foi excluído porque o desfecho não atendia aos critérios estabelecidos. Foram selecionados seis artigos. Todos os 6 estudos foram realizados 
em países de alta renda, quatro foram estudos de coorte e dois estudos de corte transversal. Há evidência que os indivíduos com percepção de justiça organizacional alta apresentaram menor risco de doença cardiovascular (infarto e acidentes vascular cerebral) e metabólica. Conclusão: Baixa justiça organizacional tem repercussões para a saúde cardiovascular e metabólica dos trabalhadores e para as organizações. Os gestores devem elaborar estratégias para promover alta justiça organizacional e, assim, mitigar os seus impactos sobre a saúde dos trabalhadores e sobre as instituições.

Descritores: Justiça organizacional; Saúde do trabalhador; Doenças cardiovasculares; Doença das coronárias; Transtornos cerebrovasculares; Hipertensão; Doenças do sistema endócrino. 


\section{ABSTRACT}

Scalabrin A. Organizational justice and negative health outcomes: a systematic review [dissertation]. São Paulo: "Faculdade de Medicina, Universidade de São Paulo"; 2021.

Background: Work stress has repercussions for the physical and/or mental health of workers. Among the conceptual models of stress at work, Organizational Justice is one of those that have been currently investigated. This model allows us to identify aspects of the work context related to the perception of justice in the distributive (resource distribution), procedural (decision making) and interactional (communication quality and interpersonal treatment) dimensions. Several studies have investigated associations between perceptions of organizational justice and health outcomes, in particular, their impacts on workers' mental health. Some studies investigated the repercussions of experiencing organizational injustice on the cardiovascular system and metabolism of workers. However, the systematization of scientific information available on this topic is still scarce. Objective: To systematically review evidence of the association between perceived organizational justice and cardiovascular and metabolic disease in adult workers. Methods: The databases investigated were MEDLINE, EMBASE, LILACS. The search strategy used five combinations for each database, which included exposure (Organizational Justice) and an outcome (coronary artery disease; cerebrovascular disease; systemic arterial hypertension; diabetes mellitus; cardiovascular disease). Eligibility criteria included original publications from observational studies (cohort, cross-sectional or case-control) published in English, Portuguese or Spanish. The selected titles were evaluated by two reviewers, in case of dissent, a third reviewer was called. Study quality was assessed using the National Institute of Health Study Quality Assessment Tools criteria. Results: 1,932 titles were identified in the investigated databases. There were 19 articles left for full evaluation, of which 12 assessed job stress using models other than organizational justice and one was excluded because the outcome did not meet the established criteria. Six articles were selected. All 6 studies were conducted in high-income countries, four were cohort studies and two were cross-sectional studies. There is evidence that individuals with a high perception of organizational justice had a lower risk of cardiovascular (infarction and stroke) and metabolic disease. Conclusion: Low organizational justice has repercussions for cardiovascular and metabolic health of 
workers and organizations. Managers must devise strategies to promote high organizational justice and thus mitigate its impacts on workers' health and on institutions.

Descriptors: Organizational justice; Occupational health; Cardiovascular diseases; Coronary disease; Cerebrovascular disorders; Hypertension; Endocrine system diseases. 


\section{LISTA DE FIGURAS E QUADROS}

\section{FIGURAS}

Figura 1- Modelo Demanda-Controle 16

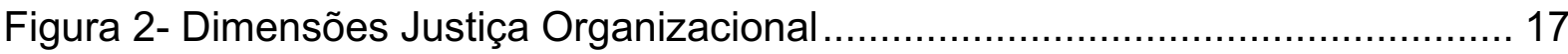

Figura 3- Ondas de desenvolvimento do modelo de Justiça Organizacional ........... 20

Figura 4- Mecanismos fisiopatológicos ligando o estresse a DCV ......................... 24

Figura 5- Seleção dos estudos- fluxograma PRISMA.............................................. 34

\section{QUADROS}

Quadro 1- Características das bases de dados utilizadas.................................... 32

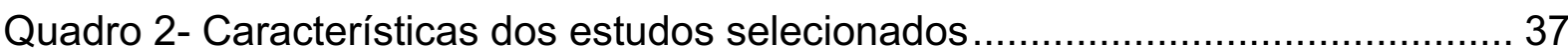

Quadro 3- Avaliação da qualidade do estudo de acordo com os critérios do Quality Assessment Tool - NIH 


\section{LISTA DE ABREVIATURAS E SIGLAS}

AHA- American Heart Association

IC- Intervalo de Confiança

IMC- Índice de Massa Corporal

NCEPIATP III - Third Report of the National Cholesterol Education

Program (NCEP) Expert Panel on Detection, Evaluation, and Treatment of High Blood Cholesterol in Adults (Adult Treatment Panel III)

$\mathrm{NIH}-$ National Institute of Health

NHLBI- National Heart, Lung and Blood Institute

OMS- Organização Mundial da Saúde

QJO- Questionário de Justiça Organizacional 


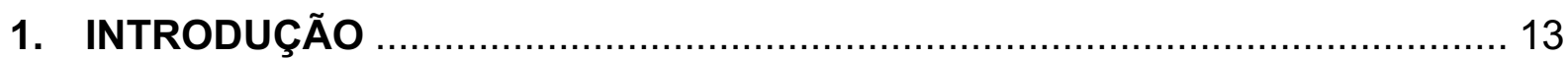

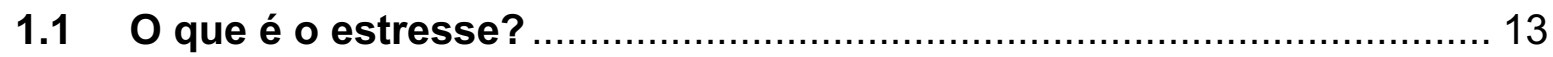

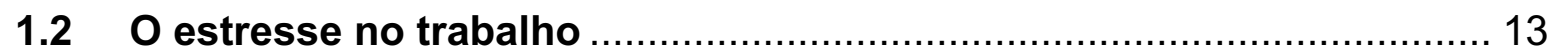

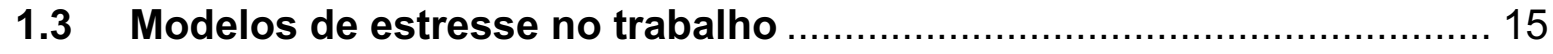

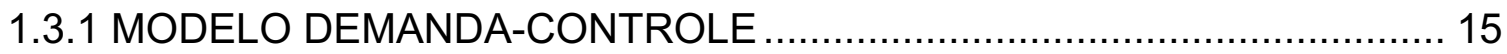

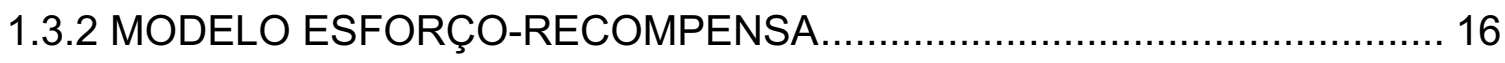

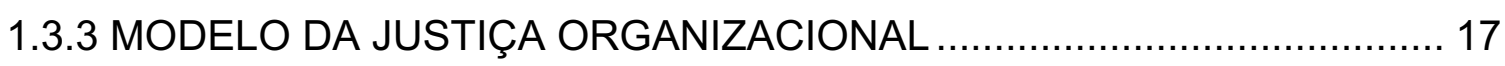

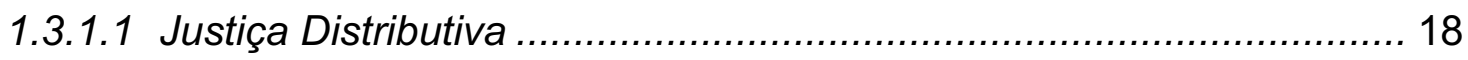

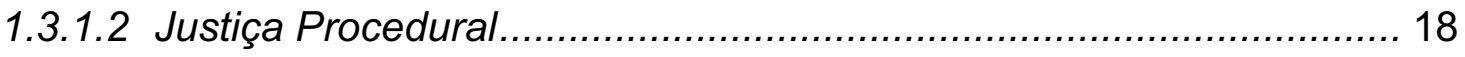

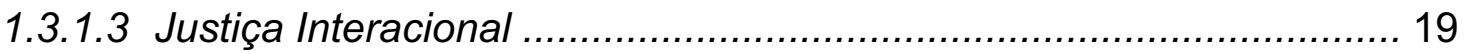

1.3.1.4 Modelo integrado de Justiça Organizacional .................................. 19

1.4 Formas de investigar Justiça Organizacional ...................................... 20

1.4.1 HISTÓRICO DO DESENVOLVIMENTO DOS INSTRUMENTOS .............. 20

1.4.2 DESAFIOS PARA A AVALIAÇÃO DE JUSTIÇA ORGANIZACIONAL ...... 22

1.5 Justiça Organizacional e Saúde …………….................................. 22

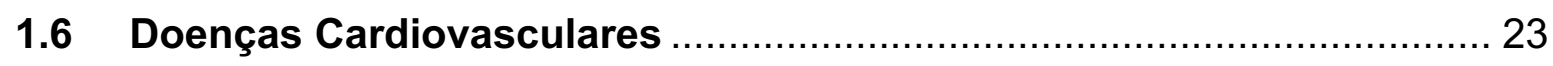

1.6.1 RESPOSTA DO ORGANISMO AO ESTRESSE ................................ 23

1.6.2 ESTRESSE NO TRABALHO E DOENÇAS CARDIOVASCULARES ....... 25

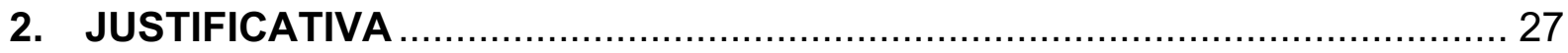

3. OBJETIVO

4. MÉTODO

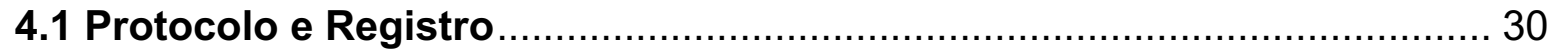

4.2 Elegibilidade: Critérios de Seleção …………..................................... 30

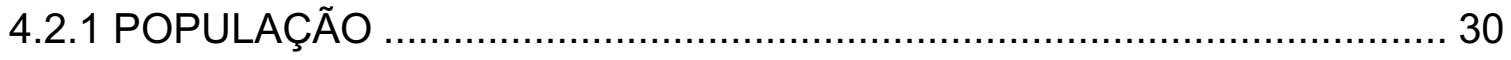

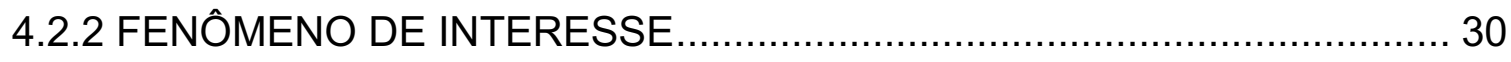

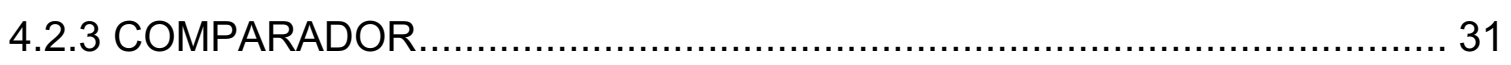

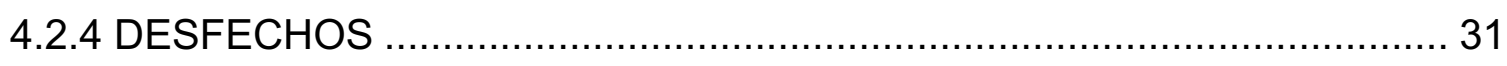

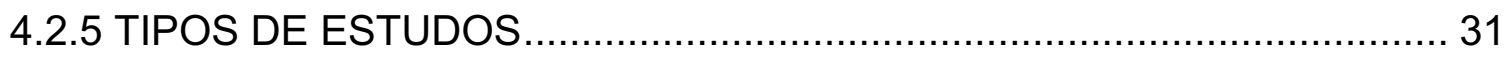




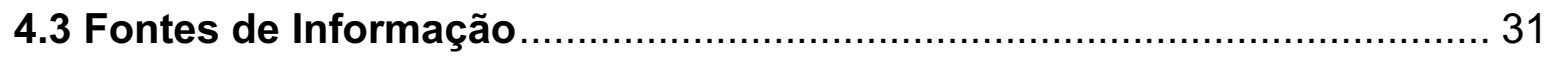

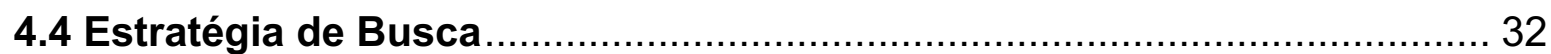

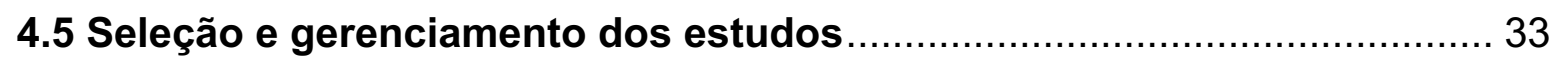

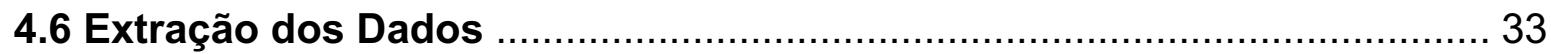

4.7 Avaliação da Qualidade dos Estudos ………........................................... 34

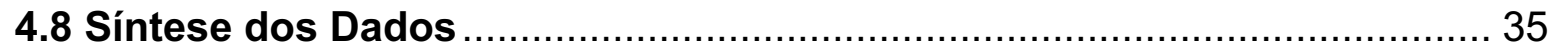

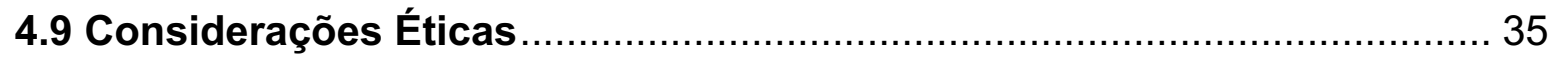

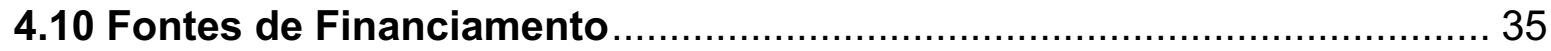

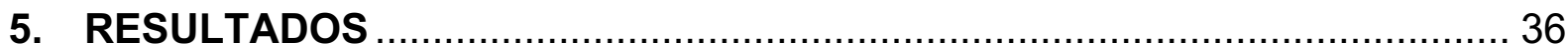

5.1 Características dos Estudos e Síntese dos Resultados .......................... 36

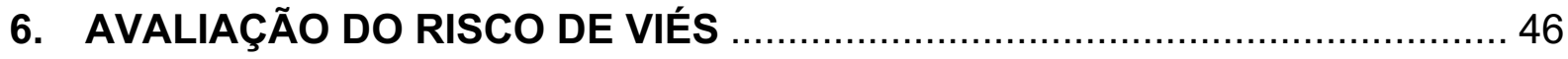

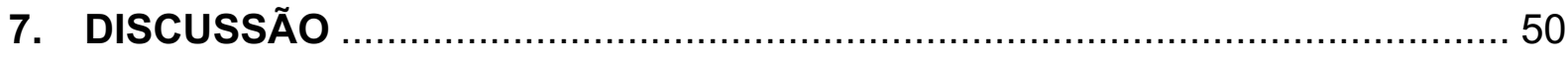

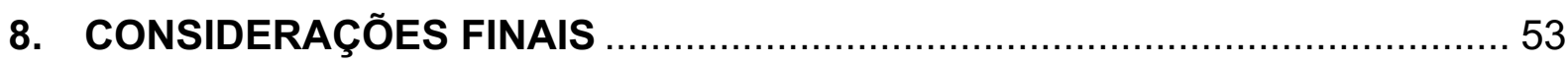

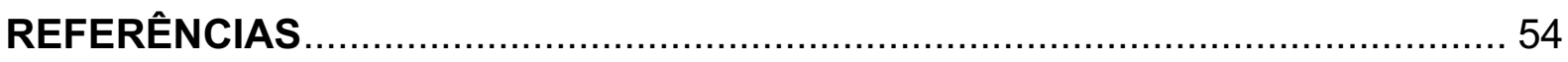

ANEXO

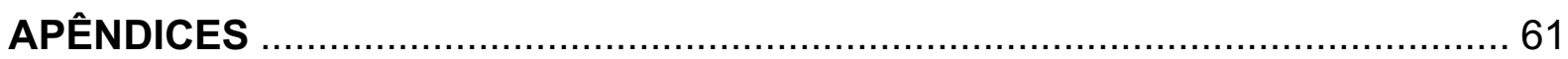

APÊNDICE A- Estratégia de Busca- PubMed ................................................ 61

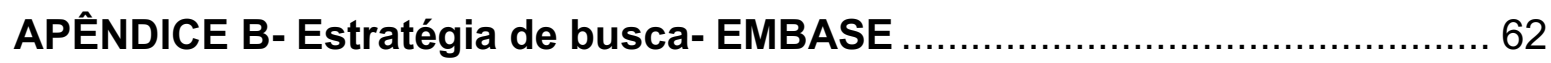

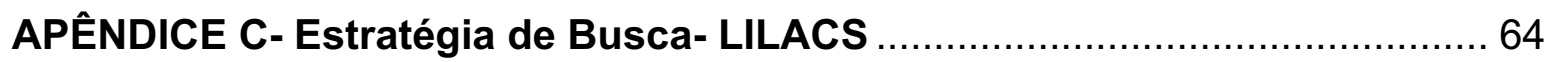




\section{INTRODUÇÃO}

\subsection{0 que é o estresse?}

O conceito de estresse, dentro do contexto da saúde, começou a ser utilizado em 1936 por Hans Selye, a partir do modelo da Síndrome de Adaptação Geral. De acordo com essa teoria, uma variedade de estímulos físicos, químicos, biológicos e psicossociais (agentes estressores) gerariam um padrão de respostas fisiológicas adaptativas em três etapas. A fase de alarme é a resposta inicial ao estresse, caracterizada pela ativação neural, que leva à rápida secreção de catecolaminas e estímulo ao eixo hipotálamo-hipófise-adrenal. Na fase seguinte, a de resistência, entram em ação os mecanismos mais lentos de adaptação, com elevação crônica de cortisol e modificação do metabolismo. Caso esses estímulos estressores sejam prolongados, frequentes ou intensos podem causar no organismo um estado de desgaste, caracterizando a fase de exaustão $(1,2)$.

Na década de 1990, McEwen explorou em diversas publicações o conceito de alostasia e carga alostática (3). Enquanto que a homeostasia se refere a um estado de equilíbrio necessário ao bom funcionamento de órgãos e tecidos, a alostasia é o processo pelo qual são realizados ajustes para obter esse equilíbrio de acordo com as demandas do ambiente (4). Os principais sistemas que promovem adaptação são o sistema nervoso autônomo e o eixo hipotálamo-hipófise adrenal. Em situação de ativação prolongada destes sistemas, em vez de obter os benefícios adaptativos, o organismo passa a ser mais suscetível a doenças, situação que caracteriza a carga alostática.

Assim como o estresse de origem física, o de origem psicossocial tem potencial de gerar danos ao organismo pelos mecanismos fisiopatológicos citados. Também podem ocorrer danos por vias indiretas, através de comportamentos mal adaptativos, como uso de tabaco e álcool, sedentarismo e dieta inadequada $(5,6)$.

\subsection{0 estresse no trabalho}

O trabalho é um elemento muito importante na vida das pessoas. Entre os aspectos psicossociais mais relevantes estão a formação de rede social, estruturação 
do tempo e a construção da identidade, além da função instrumental de provimento de recursos financeiros (7). O desemprego já foi associado a desfechos adversos, inclusive aumento de mortalidade por causas externas (incluindo suicídio) e doenças do aparelho circulatório, especialmente em homens (8-10). O desemprego foi associado a estigma social, baixa autoestima, baixa satisfação com a vida, perda de contatos sociais e consequências para a saúde mental (7).

Entretanto, ao mesmo tempo que estar sem emprego pode trazer prejuízos à saúde, estar empregado sob condições geradoras de estresse excessivo também pode fazê-lo. Há um crescente volume de evidências associando consistentemente más condições de trabalho a transtornos da saúde física e mental, tais como distúrbios metabólicos, hipertensão arterial sistêmica, doença cardiovascular, doenças musculoesqueléticas, distúrbios do sono, depressão, ansiedade e Síndrome de Burnout (11-18).

Os riscos ocupacionais são caracterizados como sendo o potencial que um agente, procedimento ou situação tem de causar dano ou efeitos adversos à saúde no contexto do trabalho. Segundo a Organização Internacional do Trabalho, os riscos (ocupacionais) psicossociais são descritos como as interações entre aspectos do trabalho (ambiente, conteúdo e condições organizacionais) com as capacidades, necessidades e cultura do trabalhador que podem influenciar sua saúde, desempenho e satisfação no emprego (11). De acordo com a Organização Mundial da Saúde (OMS), o estresse no trabalho é definido como sendo a resposta que os indivíduos podem apresentar quando as demandas e as pressões do trabalho não correspondem aos seus conhecimentos e habilidades, desafiando a sua capacidade de lidar com elas (19).

O estudo de fatores psicossociais no desenvolvimento de doenças é bastante desafiador, uma vez que não segue o modelo de uma causa bem delimitada resultando um efeito igualmente delimitado. Nas análises de sistemas complexos, um desfecho pode ocorrer em função da soma e/ou interação de diversas exposições. Além disso, uma exposição pode estar associada a múltiplos desfechos $(2,12)$.

Segundo a literatura, diversos desfechos negativos em saúde têm sido associados ao estresse no trabalho, como alterações metabólicas (20), aumento de marcadores inflamatórios $(21)$, doença cardiovascular $(6,22)$ e distúrbios do sono $(14,17)$. Foi observada associação entre justiça organizacional e frequência de transtornos de saúde mental como depressão $(18,23)$. O afastamento do trabalho 
frequente e prolongado (certificados por médicos ou não) e a percepção de má saúde auto referida também foram associados à baixos níveis de justiça (24-26).

Entre os impactos do estresse no trabalho para as organizações/instituições estão o absenteísmo, turnover, erros no trabalho e presenteísmo.

\subsection{Modelos de estresse no trabalho}

Diversos modelos foram postulados na expectativa de compreender 0 fenômeno do estresse ocupacional (2). Entre eles, há três com maior destaque na literatura.

\subsubsection{MODELO DEMANDA-CONTROLE}

Um dos mais estudados é o de Demanda-Controle (Demand-Control Model), também chamado de desgaste no trabalho (Job Strain Model) (12), no qual são levados em conta essencialmente as demandas psicológicas relacionadas ao trabalho e o grau de controle/autonomia sobre o processo. De acordo com os critérios da escala de estresse no trabalho, modelo demanda-controle, a combinação dos níveis das dimensões demanda e controle produzem quatro tipos de trabalho: baixo desgaste (baixa demanda e alto controle), passivo (baixa demanda e baixo controle), ativo (alta demanda e alto controle) e alto desgaste (alta demanda e baixo controle).

Posteriormente, foi adicionada ao modelo a dimensão do apoio social, que se refere ao suporte dado ou não por seus pares ou por seus superiores. De acordo com o modelo proposto por Karasek e Theorell em 1990, o apoio social no trabalho é importante para reduzir os efeitos negativos da alta demanda e do baixo controle, contribuindo, assim, para melhorar a saúde mental. 
Figura 1- Modelo Demanda-Controle

Modelo Demanda-Controle de Karasek

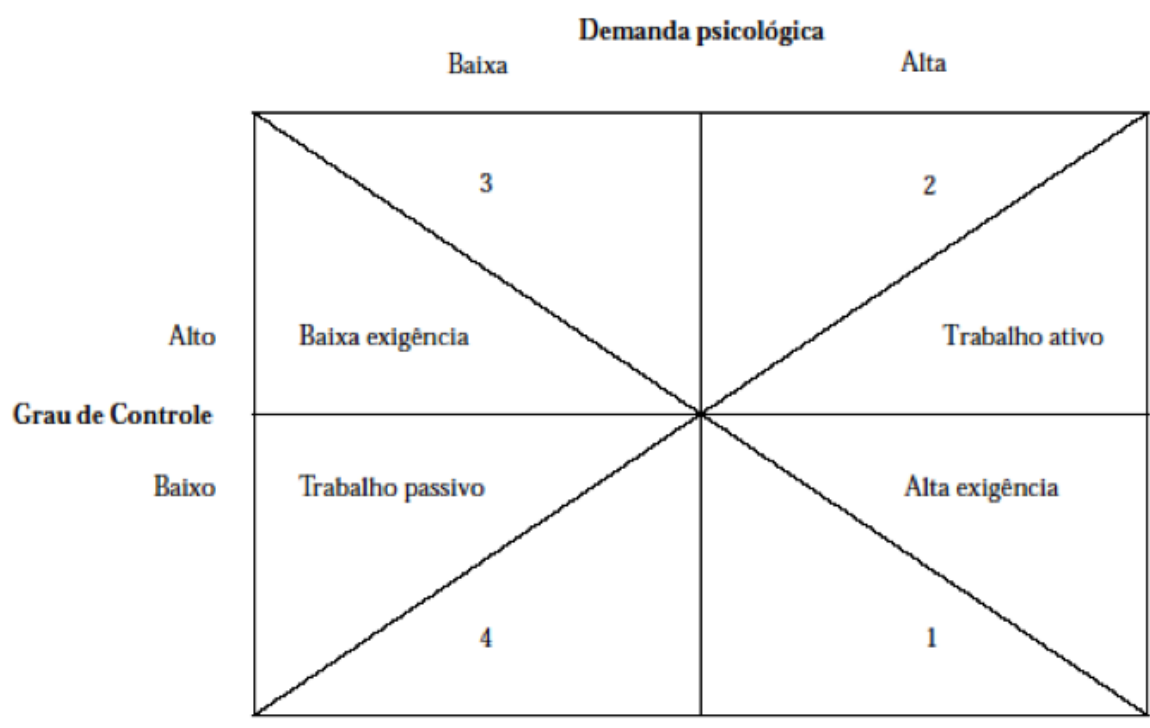

Diagonal B

Motivação para desenvolver

novos tipos de comportamento

Diagonal A

Riscos de exigência psicológica

e adoecimento psíquico.

Fonte: adaptado de Karasek \& Theorell.

Vários estudos observaram a associação entre trabalho passivo e de alto desgaste com sintomas depressivos (27-29) e com o postulado no modelo demandacontrole.

\subsubsection{MODELO ESFORÇO-RECOMPENSA}

Outro modelo bastante estudado é o modelo Esforço-Recompensa (EffortReward Imbalance Model) (30), o qual leva em consideração o equilíbrio entre o esforço realizado e a percepção de recompensa do sujeito, não só em termos monetários, mas também de reconhecimento social e oportunidades de progressão de carreira. Ambos os modelos estão diretamente relacionados com a vivência pessoal do indivíduo no seu ambiente de trabalho. 


\subsubsection{MODELO DA JUSTIÇA ORGANIZACIONAL}

Mais recentemente, vem ganhando espaço na pesquisa cientifica um terceiro modelo, o da Justiça Organizacional (Organizational Justice Model) (31,32). Este difere dos anteriores por levar em consideração o aspecto coletivo das relações de trabalho, ultrapassando a perspectiva do indivíduo (33). Ele é composto por três dimensões: distributiva, procedural (ou processual) e interacional (ou relacional). A justiça distributiva se refere a como é feita a alocação da recompensa (monetária, social ou outras) entre os trabalhadores. A justiça procedural diz respeito a como os procedimentos de tomada de decisão são realizados dentro da instituição. São considerados elementos cruciais para esta dimensão a participação nas tomadas de decisão pelas pessoas diretamente afetadas por ela, a imparcialidade dos que as tomam e a consistência no uso das regras. Também é importante que as informações utilizadas para a tomada de decisão sejam confiáveis, siga valores considerados éticos e apresente possibilidade de correção no caso de que se verifique que a ação foi inadequada. Por último, a justiça interacional se refere à maneira com a qual os superiores se relacionam com seus subordinados. O respeito, educação e consideração que são demonstrados pelas chefias colaboram para que o trabalhador sinta sua dignidade como mantida (34).

\section{Figura 2- Dimensões Justiça Organizacional}

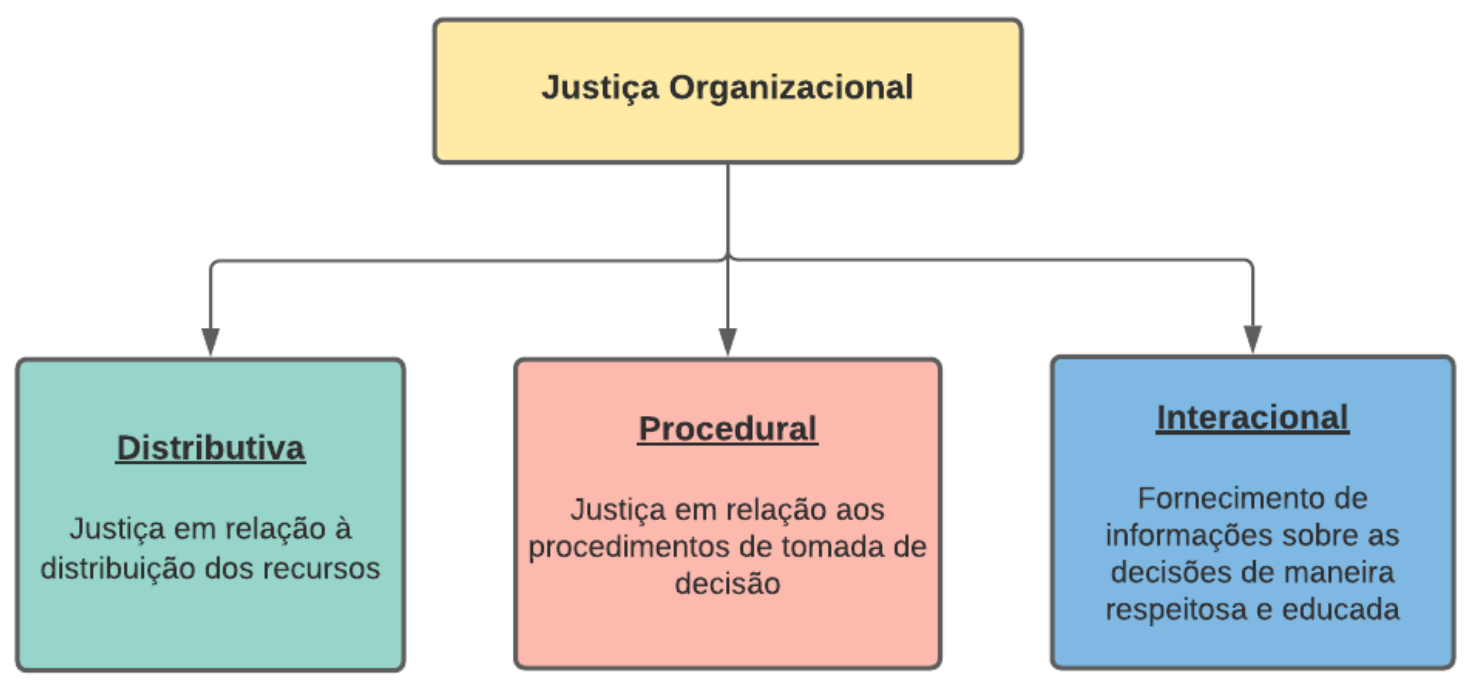


O conceito de Justiça Organizacional tal qual utilizamos atualmente, é relativamente recente. Entretanto, teve o início da sua construção há cerca de 70 anos, dentro das ciências sociais. Evoluiu em quatro etapas: onda da justiça distributiva, onda da justiça procedural, onda da justiça interacional e onda integrativa (Figura 3). Esta última reuniu as dimensões propostas anteriormente em um construto único (35).

\subsubsection{Justiça Distributiva}

A onda da Justiça Distributiva deu origem ao que é considerada a primeira dimensão da Justiça Organizacional. O conceito foi formalmente introduzido por Homans em 1961, que descreveu que os homens em uma relação de troca têm a expectativa de que o seu investimento tenha uma recompensa que seja the seja proporcional. Adams ampliou a noção de investimentos e recompensas. Para o primeiro fator, considerou elementos como educação, inteligência, experiência, treinamento, habilidades, entre outros, além do esforço efetivo do desempenho da sua função. Na retribuição, incluiu itens como pagamento, progressão de carreira, status e gratificações (34). A percepção da justiça não se dá apenas no aspecto absoluto, mas também na comparação com seus pares de forma que a alocação (ou distribuição) dos recursos é o conceito central desta dimensão.

\subsubsection{Justiça Procedural}

Posteriormente, estudos da área do direito contribuíram no desenvolvimento da segunda onda, chamada de Justiça Procedural ou Justiça Processual. Nela, foi observado que em procedimentos jurídicos havia maior percepção de justiça quando as partes tinham a oportunidade de participar ativamente do processo de resolução de disputas. Em 1980, Leventhal (36) propôs a segunda categoria de justiça, definindo como a crença que o indivíduo tem de que os procedimentos de alocação foram justos e apropriados, tendo seguido determinados critérios. Ele identificou seis regras que definiriam uma tomada de decisão como sendo justa: 
- Consistência: os procedimentos deveriam ser os mesmos, independentemente do tempo ou das pessoas envolvidas;

- Supressão de Vieses: os procedimentos não poderiam ser influenciados por interesses próprios ou baseados em preconceitos;

- Acurácia: as informações utilizadas para tomada de decisão deveriam ser o mais corretas possível;

- Possibilidade de Correcão: deveria haver a possibilidade de recorrer das decisões e corrigi-las, quando demonstrassem ser equivocadas;

- Representatividade: os procedimentos deveriam levar em conta os conceitos e opiniões das pessoas que seriam afetadas pelas decisões;

- Ética: os valores morais e éticos dos envolvidos deveriam ser respeitados.

Observou-se que quando os indivíduos percebem os procedimentos como justos há uma melhora da aceitação dos resultados, mesmo quando thes são desfavoráveis (35).

\subsubsection{Justiça Interacional}

Além de aspectos estruturais das tomadas de decisão, a qualidade da comunicação e tratamento interpessoal também é importante para os sujeitos. Baseado neste princípio se desenvolveu a Justiça Interacional ou Justiça Relacional (terceira onda) (37). Essa dimensão postula que as pessoas acreditam que é necessário receber as informações de maneira apropriada, digna e respeitosa. Alguns estudiosos do campo da Justiça Organizacional, como Greenberg e Colquitt, argumentam que esta dimensão seria composta por dois componentes: interpessoal e informacional. Enquanto na primeira, o enfoque é na forma como os superiores demonstram respeitar os sentimentos dos subordinados, na segunda, o que se busca apreciar é se as informações sobre os procedimentos de tomada de decisão são repassadas de maneira clara e completa. Entretanto, há estudos demonstrando que esses dois componentes são altamente correlacionados e a maior parte das pesquisas tendem a considerar como uma dimensão única (35).

\subsubsection{Modelo integrado de Justiça Organizacional}


Por fim, na quarta onda, cujo início é difícil de determinar, passa-se a estudar os efeitos em combinação das três dimensões citadas previamente. A onda integrativa resultou no conceito como é majoritariamente utilizado no presente. O campo de estudo da Justiça Organizacional ainda está em desenvolvimento. Existe uma aceitação geral do conceito de que ela pode ser entendida como o quanto um aspecto do ambiente organizacional é percebido como justo de acordo com certas regras ou padrões. Na tentativa de compreender e explicar o fenômeno, é possível observar o surgimento de múltiplos novos constructos, demonstrando que ainda há necessidade de amadurecimento dessa área de estudo (38).

Figura 3- Ondas de desenvolvimento do modelo de Justiça Organizacional

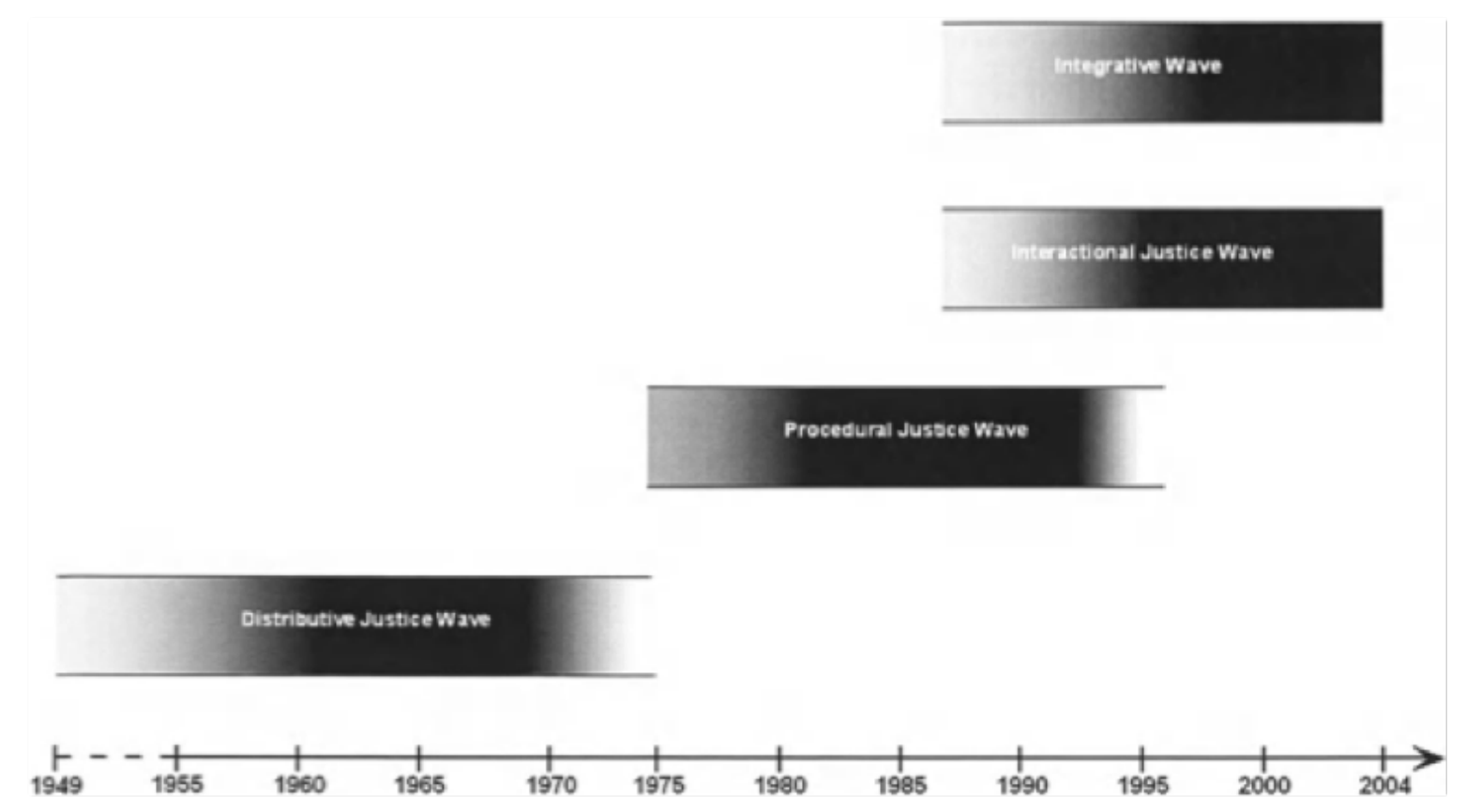

Fonte: Handbook of Organizational Justice (Greenberg \& Colquitt 2005).

\subsection{Formas de investigar Justiça Organizacional}

\subsubsection{HISTÓRICO DO DESENVOLVIMENTO DOS INSTRUMENTOS}


Em fases iniciais da construção do conceito de justiça organizacional, houve um momento em que as teorias avançavam, mas sem haver, entretanto, maneiras fidedignas de mensurá-las. Foi apontado por Greenberg (31) que, naquele período, a maioria dos pesquisadores utilizava questionários de apenas um item (por vezes dois ou três), criado para aquele estudo em específico e sem demonstrar a evidência da validade do constructo. Nas décadas de 1970 e 1980, a investigação das percepções de justiça ocorria principalmente em cenários de simulação em estudos acadêmicos da área do direito, geralmente por meio de perguntas como "quão justo" determinado evento seria. Havia pouca distinção se os objetos em questão eram referentes a decisões de pessoas ou a sistemas formais de procedimentos. Estudos investigando utilização de múltiplas questões se tornaram mais comuns no final da década de 1980, quando se passou a incluir perguntas que avaliavam diretamente a percepção de justiça em relação a atitudes de pessoas (39).

Em 1986, Price e Mueller (40), ao avaliar absenteísmo e intenção de deixar o emprego em um hospital, criaram o Índice de Justiça Distributiva, que foi incorporado como um dos principais instrumentos para avaliar a dimensão distributiva e é utilizado até hoje. Trata-se de um questionário composto por seis perguntas, cuja reposta é dada dentro de uma escala Likert de cinco pontos.

Em 1991, Moorman (32) publicou um estudo que investigou a relação percepção de justiça e sua associação com comportamentos e atitudes dos empregados. O questionário formulado na ocasião, que sofreu pequenos ajustes posteriormente (41) é, até os dias atuais, um dos mais utilizados. O chamado Questionário de Justiça Organizacional (QJO) foi um marco neste campo de estudo. Ele trazia como novidade conter não só o componente interacional, que observava a relação dos supervisores com os seus subordinados, mas também incorporou a investigação dos procedimentos formais adotado pelas organizações. Também tinha um aspecto mais abrangente, saindo e um enfoque episódico, que avaliava eventos específicos, para um enfoque sistêmico. Apesar de ter sido desenvolvido em língua inglesa no começo dos anos 1990, foi apenas na década seguinte que se pôde verificar a expansão de suas validações em outros idiomas/países. O Brasil foi um dos primeiros a apresentar uma validação do QJO, no ano de 2003 (42). Países como Japão e Turquia só tiveram suas versões em 2009, Alemanha em 2015 e Portugal em 2020. Possivelmente este fator contribua para uma menor variedade entre os países 
que publicam sobre o assunto, em especial, ainda são poucos os estudos longitudinais.

O instrumento mais recente para avaliação de justiça foi criado por Colquitt em 2001 (43). Uma das principais diferenças em relação ao QJO é o fato de a dimensão interacional ser avaliada dividida nos seus aspectos interpessoal e informacional. Apesar de ser possível encontrar estudos que utilizaram esse instrumento, ele é utilizado em proporção muito menor que o questionário de Moorman.

\subsubsection{DESAFIOS PARA A AVALIAÇÃO DE JUSTIÇA ORGANIZACIONAL}

Como o campo de estudo da Justiça Organizacional é relativamente recente e o desenvolvimento do seu constructo ainda é tema de debate, não surpreende haver muita heterogeneidade nas pesquisas. Podem ser encontrados estudos mais antigos que avaliaram através de pergunta única, outros com questionários próprios e outros que utilizaram diferentes instrumentos validados. Além disso, nem todos os estudos optam por avaliar as três dimensões da Justiça Organizacional. É possível encontrar avaliações de uma única dimensão, diferentes combinações de duas dimensões e alguns com todas as três. Essas peculiaridades dificultam a comparação entre os achados dos estudos e a construção de um corpo de conhecimento consistente a respeito do tema. Entretanto, na medida em que se consolidam instrumentos validados, pode-se esperar que nos próximos anos as publicações adotem linhas de investigação mais homogêneas.

\subsection{Justiça Organizacional e Saúde}

Por um longo período de tempo, a pesquisa sobre Justiça Organizacional ficou restrita aos estudos da área de psicologia organizacional, administração e recursos humanos, investigando sua relação com satisfação no trabalho, comprometimento organizacional, intenção de deixar o trabalho e absenteísmo, entre outros (44-46).

A intersecção com a saúde passou a acontecer nas duas últimas décadas, a partir da publicação de Elovainio (41), que identificou associação entre injustiça risco aumentado para má saúde, transtornos psiquiátricos menores e ausência do trabalho por motivo de doença. 


\subsection{Doenças Cardiovasculares}

Doenças Cardiovasculares (DCV) correspondem a um grupo de patologias do coração e dos vasos sanguíneos (47). Entre os seus principais tipos estão as doenças arteriais coronarianas (angina pectoris, infarto) e doenças cerebrovasculares (acidente vascular encefálico). Conforme o Global Burden of Disease Study (48) de 2017, essas condições foram respectivamente a primeira e a terceira causas de morte no mundo, demonstrando ser um problema de grande relevância dentro da saúde pública. Em conjunto, o grupo de doenças cardiovasculares foi responsável por $31 \%$ de todos os óbitos globalmente (47). Níveis elevados de pressão arterial e glicose sanguínea são considerados importantes fatores de risco para DCV. Quando considerados ambos os sexos, estão entre os três principais fatores de risco para morte e incapacidade prematuras (48).

O desenvolvimento das doenças do sistema cardiovascular é de origem multifatorial. Alguns fatores de risco já são fartamente estudados e bem estabelecidos na literatura, sendo que dieta inadequada, sedentarismo, colesterol elevado, excesso de peso e pressão arterial aumentada podem explicar cerca de $75 \%$ da incidência de doença arterial coronariana. Deste modo, há uma parcela considerável de fatores que ainda não tem seu papel completamente esclarecido na fisiopatologia deste grupo de doenças. Existem diversos fatores sendo estudados, como componentes hormonais, mediadores inflamatórios, antecedentes perinatais, fatores socioeconômicos e estresse psicossocial (49).

\subsubsection{RESPOSTA DO ORGANISMO AO ESTRESSE}

Quando um organismo é submetido ao estresse, produz uma resposta neuroendócrina com dois componentes principais: o sistema nervoso autônomo e o eixo hipotálamo-hipófise-adrenal. O sistema nervoso autônomo, composto pelo simpático e parassimpático, mantem um tônus basal, podendo realizar ajustes momento a momento. Sua ativação ocorre através de estímulos centrais, pelo hipotálamo, tronco cerebral e medula espinhal e estímulos periféricos, através dos reflexos viscerais. Quando o organismo é submetido a um evento estressor, quase instantaneamente há uma ativação neural em massa do sistema nervoso simpático, 
com a liberação de noradrenalina. Essa resposta é amplificada quando as terminações nervosas na medula adrenal estimulam a liberação de adrenalina e noradrenalina. O mecanismo humoral traz como vantagem uma resposta mais sustentada, além da possibilidade de interagir com células que não recebem inervação direta. Com a ativação simpática, observa-se aumento da frequência e da contratilidade cardíaca e vasoconstrição na maior parte das artérias do corpo. Desta forma, obtém-se um maior débito cardíaco e aumento da pressão arterial, com priorização do fluxo sanguíneo para o sistema nervoso e para os músculos esqueléticos, num estado chamado de luta ou fuga (50).

Figura 4- Mecanismos fisiopatológicos ligando o estresse a DCV.

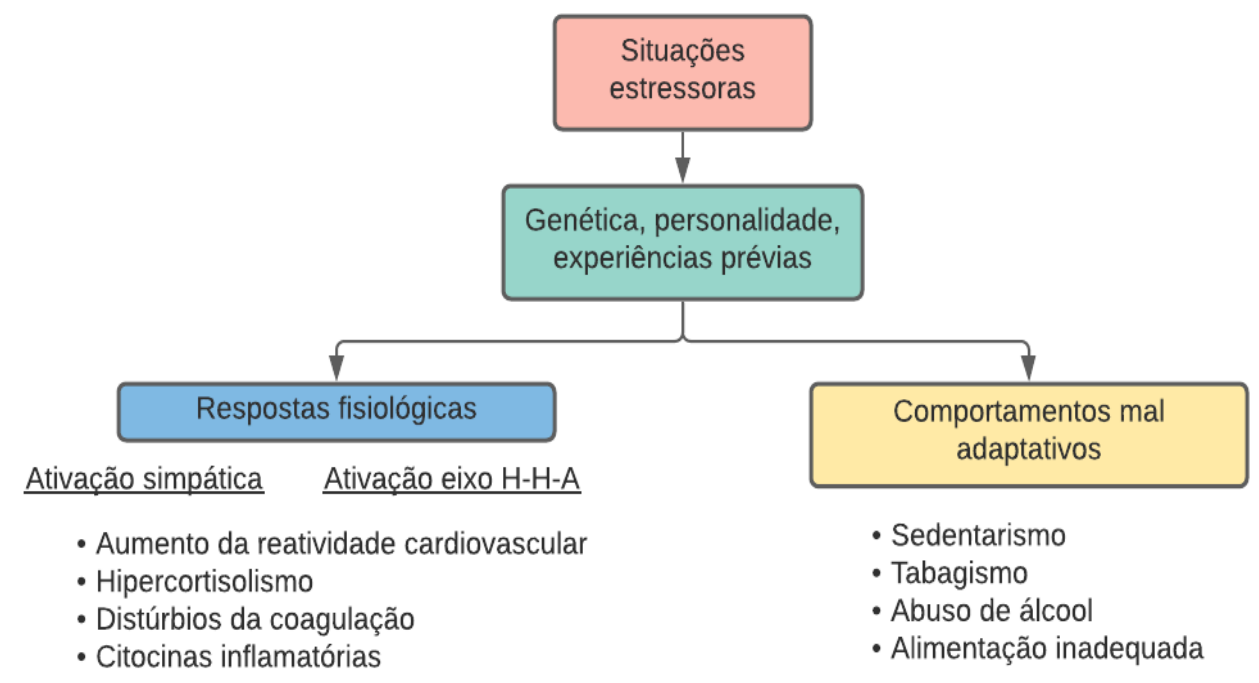

Fonte: Elaborado pela autora. (Eixo H-H-A: hipotálamo-hipófise-adrenal)

Durante uma situação de estresse também há uma ativação do eixo hipotálamo-hipófise-adrenal, através da secreção de hormônio liberador de corticotrofina $(\mathrm{CRH})$ pelo hipotálamo, que por sua vez, promove a liberação do hormônio adrenocorticotrófico (ACTH) pela hipófise anterior. No córtex adrenal, o ACTH estimula a produção e a liberação de cortisol. Este hormônio tem diversas funções, com destaque no metabolismo energético. No intuito de manter a glicose disponível para uso imediato, o cortisol promove glicogenólise no fígado, ao mesmo tempo que mobiliza ácidos graxos e aminoácidos para a gliconeogênese. Além disso, 
promove uma resistência periférica à ação da insulina, diminuindo a utilização celular de glicose pela maioria das células, privilegiando tecidos como o sistema nervoso, cuja fonte primária de energia é a glicose e não depende de insulina para sua utilização. Em condições normais, o aumento dos níveis de cortisol provoca um feedback negativo sobre o hipotálamo e a hipófise anterior, reduzindo a secreção tanto do $\mathrm{CRH}$, como do ACTH. Entretanto, esse mecanismo é prejudicado quando a produção de cortisol é excessiva e prolongada, de modo a perpetuar o processo (50).

\subsubsection{ESTRESSE NO TRABALHO E DOENÇAS CARDIOVASCULARES}

Uma das explicações mais aceitas para a ligação do estresse crônico e danos à saúde é a hipótese da alostasia/carga alostática. Enquanto que a alostasia pode ser entendida como os processos utilizados pelo organismo para retornar à situação basal de equilíbrio (homeostasia), a carga alostática representa o estado de desgaste (wear and tear) pela ativação excessiva deste mecanismo, quando em vez de trazer os benefícios adaptativos, acaba facilitando o aparecimento de doenças (3,51).

Em um organismo saudável, existe um equilíbrio entre o tônus simpático e o tônus parassimpático (ou vagal), que pode ser identificado, entre outras coisas, pela presença variabilidade (em milissegundos) entre um batimento cardíaco e outro. Quando há um excesso de atividade simpática, pode-se observar uma redução de variabilidade, que está associado a risco cardiovascular aumentado (52). Uma revisão sistemática de 2010, que avaliou os três principais modelos de estresse no trabalho (Demanda-Controle, Esforço-Recompensa e Justiça Organizacional) encontrou nos três casos associação com tônus parassimpático (vagal) diminuído, demonstrando um desequilíbrio do Sistema Nervoso Autônomo (53).

Em 2006, foi realizada uma revisão sistemática com estudos prospectivos para avaliar a incidência de doença arterial coronariana relacionada ao estresse psicossocial laboral a partir dos modelos já descritos anteriormente. Foi identificado um excesso de risco de $50 \%$ para doença cardiovascular em empregados com estresse no trabalho. Na ocasião os autores chamaram a atenção à heterogeneidade na forma de avaliar os três modelos de estresse e o fato de haver poucos dados em relação ao risco em mulheres. Dos 14 estudos avaliados, apenas um era referente a Justiça Organizacional (54). 
Em 2018, houve a publicação de nova revisão sistemática que investigou estressores do trabalho com incidência de doença arterial coronariana (22) a partir de estudos prospectivos. Sua pesquisa restringiu a busca a apenas uma base (MEDLINE). Foram identificados 23 estudos, dos quais 17 avaliaram pelo modelo Demanda-Controle apenas, 2 pelo Esforço- Recompensa apenas, 2 avaliaram ambos os modelos (separadamente) e 2 avaliaram Justiça Organizacional. Dos 23 estudos, 11 não encontraram associação significativa com entre estresse no trabalho e doença arterial coronariana. Entre os 12 que encontraram, o risco relativo variou entre 1,22 $(1,01-1,46)$ e $4,53(1,43-14,3)$. Os autores exploram as dificuldades em realizar comparações entre os estudos, devido às grandes variações em termos de tamanho de amostra, tempo de acompanhamento e definições das exposições e dos desfechos. Outro aspecto levantado é que esses modelos de estresse geralmente tem os indivíduos caracterizados dentro de medianas, tercis e quartis, de modo que a definição de quem está com estresse aumentado vai depender também dos demais indivíduos da amostra. Eles concluem que parece haver este vínculo, porém o papel do estresse no trabalho ainda necessita de mais esclarecimentos. 


\section{JUSTIFICATIVA}

O trabalho é um componente extremamente importante na vida do indivíduo e para a sociedade como um todo. Nos cenários de trabalho percebidos como injustos, os reflexos na saúde trazem consequências graves para o trabalhador e para as instituições.

A associação entre estresse no trabalho e doença cardiovascular está mais bem estabelecida com a utilização dos dois primeiros modelos- Demanda-Controle e Esforço-Recompensa, enquanto no terceiro modelo- Justiça Organizacional, ainda que venha seguindo a mesma tendência, não há o mesmo volume de conhecimento acumulado. Kasl (55) observa que neste campo de estudo, o sucesso de um modelo explanatório poderia pressupor a inadequação de outro. Entretanto, ele alerta que mais provavelmente modelos diversos atuam de maneira complementar, na medida em que ajudam: a promover uma compreensão maior de contexto e identificar potenciais antecedentes; a identificar processos mediadores; a oferecer variáveis com poder explanatório independente adicional.

As revisões sistemáticas anteriores abordaram apenas o desfecho de doença arterial coronariana e utilizaram apenas uma base de dados. Esta revisão se propõe a explorar outros desfechos relacionados à Justiça Organizacional. As buscas incluem a utilização de outras bases, incluindo as da América Latina.

Além de melhorar o entendimento das repercussões na saúde relacionadas ao estresse no trabalho, uma das principais razões para investigar os diferentes modelos é a possibilidade de desenvolver intervenções mais adequadas e efetivas. Sendo a justiça organizacional um fator potencialmente modificável, merece atenção dos estudos, de modo que possam ser elaboradas intervenções. Além do bem-estar físico e emocional dos sujeitos, também tem impacto em termos de afastamento curto ou prolongado do trabalho. Pode haver reflexos na manutenção da produtividade, não só na iniciativa privada, mas também nos serviços públicos, que atendem aos interesses das populações. Além disso, potencialmente pode reduzir a carga do sistema previdenciário, já sobrecarregado. Assim, identificar fatores associados à justiça organizacional torna-se fundamental para trabalhadores e gestores.

Tradicionalmente, as proposições de intervenção para diminuir o sofrimento pelo estresse entre os trabalhadores têm foco no indivíduo, como terapia cognitivocomportamental, terapia de relaxamento, grupos de apoio emocional e meditação 
$(56,57)$. Entretanto, tem sido demonstrado que o melhor resultado ocorre com a combinação de intervenções com foco tanto no indivíduo, como na organização e que, quando apenas uma estava presente, a intervenção organizacional era mais importante (58). Por esta razão, o papel do modelo da justiça organizacional deve ser mais explorado, na perspectiva de que melhores estratégias de enfrentamento possam ser desenvolvidas. 


\section{OBJETIVO}

Revisar sistematicamente evidências de associação entre níveis de Justiça Organizacional e doença cardiovascular, hipertensão arterial sistêmica e diabetes mellitus em trabalhadores adultos. 


\section{MÉTODO}

Este estudo foi confeccionado a partir das diretrizes metodológicas estabelecidas no guia do Centre for Reviews and Dissemination (CDR)- CDR's guidance for undertaking reviews in healthcare da Universidade de York (59).

\subsection{Protocolo e Registro}

O protocolo foi redigido obedecendo às orientações contidas no PRISMA-P Statement- Preferred Reporting Items for Systematic Review and Meta-Analysis Protocols (60).

Esta revisão foi registrada na base de dados PROSPERO- International Prospective Register of Systematic Reviews sob o código de identificação CRD42020215322. O registro nesta base tem como finalidade disponibilizar a informações de que revisões estão em andamento para evitar duplicidade de investigações. Além disso, ele aumenta a transparência do processo, uma vez que explicita os critérios utilizados para a realização da pesquisa e se houve qualquer modificação durante a sua execução.

\subsection{Elegibilidade: Critérios de Seleção}

\subsubsection{POPULAÇÃO}

Trabalhadores adultos homens e mulheres, empregados em instituições públicas ou privadas de qualquer ramo produtivo. Não foram feitas restrições ao perfil do local (países de quaisquer níveis de renda/desenvolvimento) nem ao tipo de atividade desenvolvida (especializada ou não especializada).

\subsubsection{FENÔMENO DE INTERESSE}

O fenômeno de interesse avaliado é a percepção de baixa Justiça Organizacional, mensurada, preferencialmente, através de questionários quantitativos validados. Não foram excluídos estudos que utilizaram outros instrumentos, porém foi levado em consideração na discussão dos resultados. 


\subsubsection{COMPARADOR}

O comparador é a percepção de níveis elevados de Justiça Organizacional.

\subsubsection{DESFECHOS}

Os desfechos definidos foram os seguintes: doença cardiovascular, doença arterial coronariana, doença cerebrovascular, hipertensão arterial sistêmica e diabetes mellitus. Não foram excluídos artigos em função do método de aferição dos desfechos. Entretanto, estas informações serão apontadas nas descrições dos resultados.

\subsubsection{TIPOS DE ESTUDOS}

Foram incluídos nos critérios de busca estudos observacionais transversais, coortes ou de caso-controle. Utilizou-se apenas artigos com informações originais. Revisões sistemáticas ou narrativas foram excluídas da pesquisa, porém foram utilizadas para verificação manual de referências. Não foram feitas restrições em termos de tamanho da amostra ou período de acompanhamento. Nesta pesquisa nenhuma data limite foi imposta. Foram considerados para avaliação artigos publicados em Inglês, Português ou Espanhol.

\subsection{Fontes de Informação}

A revisão sistemática da literatura foi realizada através da busca nas seguintes bases de dados: Medical Literature Analysis and Retrieval System Online (MEDLINE) via PubMed, Excerpta Medica Database (EMBASE), Literatura Latino-Americana e do Caribe em Ciências da Saúde (LILACS) (Quadro 1). Além disso, foi realizada busca complementar através nas listas de referências dos artigos identificados, revisões, meta-analises e capítulos de livros. 
Quadro 1- Características das bases de dados utilizadas.

\begin{tabular}{|c|c|c|}
\hline Nome da Base & Características & Forma de Acesso \\
\hline $\begin{array}{l}\text { Medical Literature Analysis and Retrieval } \\
\text { System Online (MEDLINE) }\end{array}$ & $\begin{array}{l}\text { Base de dados especializada em publicações } \\
\text { biomédicas em periódicos científicos }\end{array}$ & $\begin{array}{l}\text { Acesso gratuito através do site: } \\
\text { https://pubmed.ncbi.nlm.nih.gov }\end{array}$ \\
\hline Excerpta Medica Database (EMBASE) & $\begin{array}{l}\text { Base de dados da área da biomedicina e } \\
\text { farmacologia, inclui artigos científicos e } \\
\text { trabalhos de eventos }\end{array}$ & $\begin{array}{l}\text { Acesso pago através do site: } \\
\text { https://www.embase.com }\end{array}$ \\
\hline $\begin{array}{l}\text { Literatura Latino-Americana e do Caribe } \\
\text { em Ciências da Saúde (LILACS) }\end{array}$ & $\begin{array}{l}\text { Base de dados da área da saúde, inclui artigos } \\
\text { científicos, relatórios técnicos, teses e } \\
\text { dissertações especializada nas publicações da } \\
\text { América Latina e Caribe. }\end{array}$ & $\begin{array}{l}\text { Acesso gratuito através do site: } \\
\underline{\text { https://lilacs.bvsalud.org }}\end{array}$ \\
\hline
\end{tabular}

Fonte: Elaborado pela autora.

A busca dentro da literatura cinzenta se ficou delimitada às teses e dissertações disponíveis na plataforma da Coordenação de Aperfeiçoamento de Pessoal de Nível Superior (CAPES) do Ministério da Educação do Brasil.

A realização das buscas ocorreu em 04 de agosto de 2020. Foi realizada uma atualização em 20 de fevereiro de 2021, para confirmar se nenhum outro estudo relevante havia sido incluído nas bases de dados.

\subsection{Estratégia de Busca}

A estratégia de busca foi elaborada pela pesquisadora principal (Angela Scalabrin) com a colaboração de uma bibliotecária. Ela foi construída através de palavras chave, sinônimos e variações de grafia da exposição e de cada desfecho. Foram utilizados também os vocabulários controlados de cada base, também conhecidos como descritores de assunto. No PubMed os termos chamam-se MeSH (Medical Subject Headings), enquanto que no EMBASE têm-se o Emtree. Por sua vez, a Base LILACS utiliza DeCS (Descritores em Ciências da Saúde).

Foram montadas 5 combinações para cada base, que incluíam a exposição (Justiça Organizacional) e um desfecho (Doença arterial coronariana; Doença 
cerebrovascular; Hipertensão Arterial Sistêmica; Diabetes Mellitus; Doença cardiovascular), utilizando os operadores booleanos "OR” e "AND”. As estratégias estão reproduzidas no apêndice. Na tentativa de aumentar a sensibilidade, não utilizamos os filtros das bases, para evitar perder artigos por problemas de indexação.

\subsection{Seleção e gerenciamento dos estudos}

Duas revisoras (Angela Scalabrin e Andréa Tenório Correia da Silva) atuaram de maneira independente no levantamento de estudos. A partir dos critérios definidos, foram recuperadas 2.362 citações através das bases e 8 a partir de buscas manuais (figura 5). Utilizando o software Rayyan $®$, as autoras identificaram 438 duplicatas. Após sua remoção, 1.932 publicações foram avaliadas com base no título e no resumo, resultando em 19 artigos para avaliação na íntegra. Entre estes, 12 apresentavam outros modelos de estresse no trabalho que não justiça organizacional e 1 foi excluído em função de o desfecho não corresponder aos critérios estabelecidos. Os casos de discordância foram discutidos e resolvidos por consenso. Ao final, restaram 6 estudos para serem avaliados.

\subsection{Extração dos Dados}

As duas revisoras (AS e ATCS) extraíram de maneira independente os dados através de formulário elaborado no Microsoft Excel ${ }^{\circ}$, onde foram inseridas as seguintes informações:

- Identificação do estudo: título, autores, ano de publicação, país, possíveis conflitos de interesse;

- Caracterização do estudo: desenho, duração, tamanho da amostra, taxa de participação, taxa de seguimento;

- Participantes: idade, sexo, etnia;

- Exposição: tipo de exposição avaliada (quais dimensões de justiça organizacional), instrumento utilizado para caracterizar a exposição, tipo de trabalho/organização;

- Covariáveis: descrição de quais covariáveis foram verificadas

- Desfecho: qual desfecho, maneira de aferição; 
- Resultados: estimativas de efeito, ajustes, avaliação da qualidade metodológica.

Figura 5- Seleção dos estudos- fluxograma PRISMA.

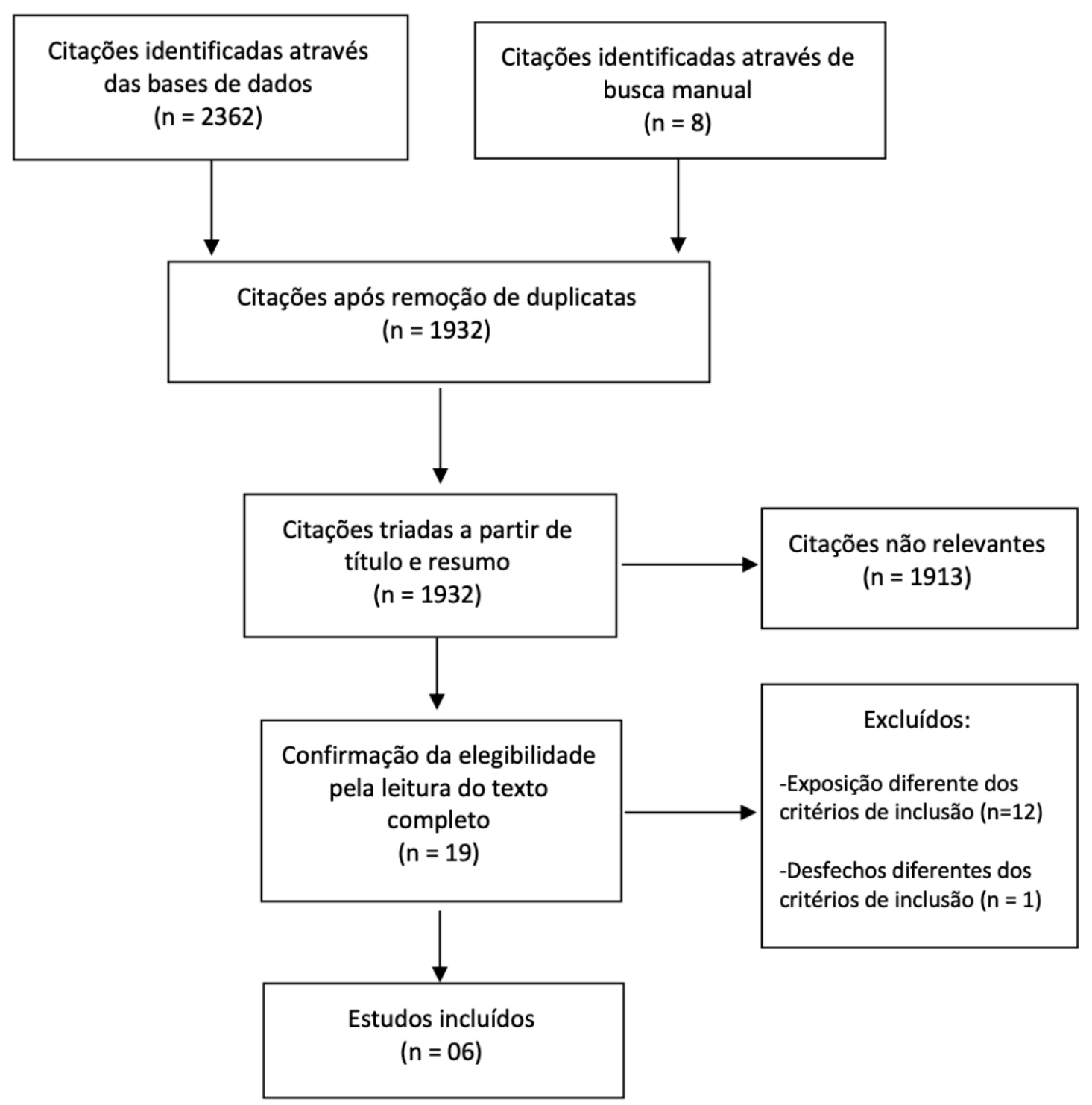

\subsection{Avaliação da Qualidade dos Estudos}

A qualidade metodológica dos estudos foi avaliada pela ferramenta desenvolvida pelo National Institute of Health (NIH-Study Quality Assessment Tools). 
Uma das vantagens desta ferramenta é que existem questionários específicos para cada tipo de desenho de estudo. As perguntas têm objetivo de identificar aspectos metodológicos ou de execução que possam comprometer a qualidade da evidência gerada, como vieses de seleção, confundimento, poder do estudo, entre outros.

O questionário desenvolvido para avaliar estudos de coorte e transversais é composto por 14 perguntas. A cada item, o pesquisador deve responder com "sim", "não" ou "não foi possível determinar/não foi relatado/não se aplica". Ao final, os estudos são classificados como com qualidade "boa", "razoável" ou "ruim". (61).

\subsection{Síntese dos Dados}

Os dados encontrados nos estudos foram apresentados através de tabelas, descrição textual e síntese narrativa.

\subsection{Considerações Éticas}

O presente estudo utilizou apenas dados publicados na literatura. Não foram usadas informações confidenciais, institucionais ou pessoais, nem tampouco foram conduzidas entrevistas. O projeto foi aprovado pelo Comitê de Ética em Pesquisa da Faculdade de Medicina da Universidade de São Paulo (Anexo A).

\subsection{Fontes de Financiamento}

Não houve fontes de financiamento para a realização desta pesquisa. 


\section{RESULTADOS}

\subsection{Características dos Estudos e Síntese dos Resultados}

No quadro 2 estão descritas as características dos estudos selecionado para a atual revisão. Os artigos identificados nesta revisão são todos provenientes de países industrializados e de alta renda (Reino Unido, Finlândia, Japão e Estados Unidos) e foram publicados entre 2005 e 2017. As amostras eram majoritariamente compostas por homens (variando de 67 a 100\%) e as médias de idade variaram entre 39 a 45 anos. Dos seis artigos, apenas três indicaram raça/cor e apresentavam em torno de $90 \%$ de brancos.

Quanto ao desenho dos estudos, quatro eram estudos de coorte e dois eram estudos de corte transversal. Com relação ao número de participantes os estudos incluíram no mínimo 290 e no máximo 6.442 participantes. Os trabalhadores que participaram dos estudos eram servidores públicos, trabalhadores da indústria ou trabalhadores da construção civil.

A dimensão interacional da justiça foi a mais frequentemente avaliada nos estudos selecionados. Os instrumentos utilizados para investigar justiça organizacional foram: (1) o questionário de justiça auto aplicado desenvolvido para o estudo Whitehall II; (2) o questionário de Justiça Organizacional de Moorman e (3) Resposta à afirmação "meu supervisor me trata de maneira justa" (escala do tipo Likert). Com relação aos resultados, os estudos de coorte encontraram associação entre nível de justiça organizacional baixo e doença cardiometabólica. 
Quadro 2- Características dos estudos selecionados para a revisão sistemática de acordo o desenho do estudo, população investigada, exposições e desfechos examinados.

\begin{tabular}{|c|c|c|c|c|c|c|}
\hline Título do estudo & $\begin{array}{l}\text { Justice at Work and } \\
\text { Reduced Risk of Coronary } \\
\text { Heart Disease Among } \\
\text { Employees- The Whitehall } \\
\text { II Study }\end{array}$ & $\begin{array}{l}\text { Justice at work and } \\
\text { cardiovascular mortality: } \\
\text { a prospective cohort } \\
\text { study }\end{array}$ & $\begin{array}{l}\text { Effects on Blood Pressure } \\
\text { Do Not Explain the } \\
\text { Association Between } \\
\text { Organizational Justice and } \\
\text { Coronary Heart Disease in } \\
\text { the Whitehall II Study }\end{array}$ & $\begin{array}{c}\text { Justice at Work and Metabolic } \\
\text { Syndrome: the Whitehall II } \\
\text { Study }\end{array}$ & $\begin{array}{l}\text { Organizational Justice and } \\
\text { Physiological Coronary } \\
\text { Heart Disease Risk Factors } \\
\text { in Japanese Employees: a } \\
\text { Cross-Sectional Study }\end{array}$ & $\begin{array}{l}\text { The moderating effect of } \\
\text { perceived organizational } \\
\text { support on the } \\
\text { relationships between } \\
\text { organizational justice and } \\
\text { objective measures of } \\
\text { cardiovascular health }\end{array}$ \\
\hline Autores & Kivimäki et al. & Elovainio et al. & Kivimäki et al. & Gimeno et al. & Inoue et al. & Rineer et al. \\
\hline Ano & 2005 & 2006 & 2008 & 2010 & 2015 & 2017 \\
\hline $\begin{array}{l}\text { Local e População } \\
\text { investigada }\end{array}$ & $\begin{array}{l}\text { Reino Unido } \\
\text { Servidores públicos } \\
\text { (homens) }\end{array}$ & $\begin{array}{l}\text { Finlândia } \\
\text { Trabalhadores de } \\
\text { indústrias ( } 67 \% \text { homens } \\
\text { e } 33 \% \text { mulheres) }\end{array}$ & $\begin{array}{l}\text { Reino Unido } \\
\text { Servidores públicos }(70.1 \% \\
\text { homens e } 29.9 \% \text { mulheres) }\end{array}$ & $\begin{array}{l}\text { Reino Unido } \\
\text { Servidores públicos ( } 69.6 \% \\
\text { homens e } 30.4 \% \text { mulheres) }\end{array}$ & $\begin{array}{l}\text { Japão } \\
\text { Trabalhadores de indústrias } \\
\text { (80\% homens e } 20 \% \\
\text { mulheres) }\end{array}$ & $\begin{array}{l}\text { Estados Unidos da América } \\
\text { Trabalhadores da } \\
\text { construção civil ( } 89,6 \% \\
\text { homens e } 10,4 \% \text { mulheres) }\end{array}$ \\
\hline Desenho do estudo & Coorte & Coorte & Coorte & Coorte & Transversal & Transversal \\
\hline $\begin{array}{l}\text { Número de } \\
\text { participantes }\end{array}$ & 6.442 & 804 & 6.062 & 6.321 & 4.499 & 290 \\
\hline $\begin{array}{l}\text { Idade ao entrar no } \\
\text { estudo (faixa etária } \\
\text { elou média de } \\
\text { idade) }\end{array}$ & $35-55$ anos & $\begin{array}{l}\text { Grupo de justiça alta- } \\
\text { idade média } 39,1 \text { anos/ } \\
\text { grupo de justiça baixa + } \\
\text { justiça intermediária- idade } \\
\text { média } 36,4 \text { anos }\end{array}$ & 35-55 anos (média 44,0) & $\begin{array}{l}35-55 \text { anos (média em homens } \\
44,8 \text { anos e em mulheres } 45,4 \\
\text { anos) }\end{array}$ & $\begin{array}{l}\text { Média de idade em homens } \\
41,5 \text { anos e em mulheres } \\
40,9 \text { anos }\end{array}$ & Média de idade 44,8 anos \\
\hline Etnia & $93 \%$ brancos e $7 \%$ outros & Não reportada & $89,4 \%$ brancos e $10,6 \%$ outros & $91,3 \%$ brancos e $8,7 \%$ outros & Não reportada & Não reportada \\
\hline $\begin{array}{l}\text { Taxa de } \\
\text { participação }\end{array}$ & $73 \%$ & Não reportada & $73 \%$ & $73 \%$ & $\begin{array}{l}90.1 \% \text { na indústria } \mathrm{A} \text { e } 96.2 \% \\
\text { na indústria } \mathrm{B}\end{array}$ & Não reportada \\
\hline
\end{tabular}


Tempo médio de seguimento

8,7 anos

25,6 anos

Não reportada

Taxa de
seguimento

$93 \%$

Justiça interacional

Organizacional

investigada

(exposição)

Forma de avaliar a

exposição

Questionário de justiça auto aplicado desenvolvido para o estudo Whitehall II

Número de

avaliações da

exposição

$\begin{array}{ll} & \\ \begin{array}{l}\text { Desfechos } \\ \text { principais }\end{array} & \begin{array}{l}\text { Morte por doença } \\ \text { não fatal, angina } \\ \text { documentada. }\end{array}\end{array}$

Morte por doenças

cardiovasculares (doenças

cardíacas isquêmicas,

outras doenças cardíacas, Pressão arterial sistólica,

doenças

outras doenças

cardiovasculares, de

pressão arterial diastólica,

coronariana, primeiro infarto

não fatal, angina documentada.

acordo com a

Classificação Internaciona

de Doenças)
18 anos

$59 \%$

Justiça interacional

Questionário de justiça auto

aplicado desenvolvido para o

estudo Whitehall II
Não se aplica

Não se aplica

Justiça procedural e justiça

interacional

Questionário de Justiça

Organizacional (versão

japonesa do questionário de

Moorman adaptado) Mueller e questionário de Justiça Organizacional (Moorman)

Pressão arterial, glicemia,

Síndrome metabólica (de acordo com os critérios do NCEP/ATP III

Hipertensão arterial sistêmica e da AHA/NHLBI)

\section{Número de}


estado marital,

Outras variáveis

escolaridade, tipo de

trabalho, colesterol total

IMC tabagismo, hipertensão

arterial sistêmica, consumo

de álcool, atividade física,

outros modelos de estress

no trabalho

Ocupação, tabagismo,

atividade física, pressão

arterial sistólica, colestero

total, IMC, outros

estressores no trabalho

(demanda-controle,

esforço-recompensa)

Menor risco de doença

coronariana (HR 0,65; 95\%

IC- 0,47-0,89) em

participantes que

reportaram níveis de justiça

interacional altos.

A associação se manteve

após ajuste por: colesterol,

indice de massa corporal,

tabagismo, hipertensão,

consumo de álcool,

atividade física, e por

fatores estressores

relacionados ao trabalh

(modelo demanda-controle

e esforço-recompensa)

demanda-controle e 0
Menor risco de morte por

doença cardiovascular em

participantes com nível de

justiça alto (HR 0,55- IC

$95 \%$ 0,34-0,88). A

associação se manteve

após ajuste por ocupação,

tabagismo, atividade

física, pressão arterial

sistólica e IMC (HR 0,59;

IC 95\% 0,36-0,96) e após

ajuste por fatores

estressores no trabalho de

acordo com modelo

modelo esforço-

recompensa (HR 0,56; IC

$95 \%$ 0,34-0,92). Quando

ambos os ajustes foram

realizados o HR foi de

0,61 com IC 95\% 0,36-

1,00 . escolaridade, ocupação,

regime de trabalho, doença

crônicas (acidente vascular

cerebral, infarto, hipertensão,

diabetes ou dislipidemia)

estresse psicológico (escala

K6), tabagismo, uso de

álcool, atividade física, IMC

colesterol HDL, colesterol

LDL e triglicerídeos

\section{Menor risco de doença arteria} coronariana em participantes

com níveis de justiça

organizacional altos (HR 0,87-

IC $95 \%$ 0,77-0,98).

-

\section{Menor risco de desenvolver contra pressão arterial elevada em homens que reportaram}

Năo encontrou associação entre justiça procedural e

interacional e os níveis

níveis de justiça organizacion

altos (HR 0,86; IC 95\% 0,78-

pressóricos dos participantes $0,95)$. Em relação ao aumento de

glicemia não foi encontrada

associação (em homens HR

1,09; IC 95\% 0,87-1,36 e em mulheres HR 0,80; IC 95\% 0,87-

1,19). sexo, estado marital, ser responsável pelo cuidado de crianças ou idosos, número

de horas trabalhadas por

semana, tempo trabalhando na empresa, cargo

(supervisor, líder, integrante

de equipe ou outros), uso de

medicamentos anti-

hipertensivos

Não encontrou associação

entre justiça procedural e

distributiva e os níveis

pressóricos dos

participantes

IMC = Índice de massa corporal; NCEP/ATP III = Third Report of the National Cholesterol Education Program (NCEP) Expert Panel on Detection, Evaluation, and Treatment of High Blood

Cholesterol in Adults (Adult Treatment Panel III); AHA/NHLBI = American Heart Association/ National Heart, Lung and Blood Institute; HR = Hazard ratio; IC 95\% = intervalo de confiança 95\%. 
Coorte Whitehall II

Os artigos 1, 2 e 3 derivam do estudo Whitehall II, uma coorte prospectiva do Reino Unido iniciada em 1985 e que continua em andamento. Foram recrutados 10.308 indivíduos (taxa de participação de $73 \%$ ), sendo 6.895 homens e 3.413 mulheres, com idade de 35 a 55 anos, que eram funcionários públicos de 20 departamentos, trabalhadores de escritórios na cidade de Londres. A coleta de dados ocorre regularmente a cada dois a cinco anos. Em todas as fases é feita uma avaliação por questionários autoaplicados. As avaliações clínicas eram realizadas nas fases de número ímpar até a nona coleta, quando passaram a ocorrer em todas as ondas de coleta. Entre os itens do questionário estão: características demográficas, histórico familiar (de saúde e socioeconômico), doenças atuais ou prévias, hábitos de vida (dieta, atividade física, uso de álcool ou tabaco), características de personalidade, eventos estressores, características do trabalho e perfil psicossocial. Nas avaliações clínicas são coletados dados antropométricos, pressão arterial, eletrocardiograma, exames laboratoriais (perfil metabólico, função renal e hepática) entre outros. Os dados de mortalidade, incluindo a causa da morte, são coletados a partir dos registros nacionais oficiais. Atualmente, o estudo está seguindo aspectos do envelhecimento, investigando multimorbidade, declínio funcional, fragilidade, deficiência e demência (62). A coorte de Whitehall II foi criada com o objetivo principal de investigar a influência de fatores psicossociais e ocupacionais nos processos de saúde-doença e morbimortalidade (63).

Nesta coorte, a percepção de justiça no trabalho foi avaliada nas duas primeiras ondas de coleta, através de um questionário autoaplicado (inserido nos anexos), composto por cinco questões ( $\alpha$ de Cronbach $=0,72$ ) explorando aspectos interacionais da justiça organizacional, que se referem à qualidade da informação que os superiores passam aos seus subordinados e se o fazem de maneira respeitosa. Os participantes classificavam as respostas numa escala do tipo Likert de quatro pontos (nunca, raramente, às vezes, frequentemente). A pontuação obtida em cada questão era somada e resultava em um escore. Este resultado era dividido em tercis. O tercil inferior era considerado como "baixa justiça", seguido por "justiça intermediária" e o superior como "alta justiça". Quando os indivíduos haviam respondido o questionário nas duas ondas de coleta, era feita uma média dos seus 
escores para subsequente classificação. Os três artigos a seguir derivam deste estudo, sendo que as informações gerais sobre a metodologia se aplicam a todos os casos.

\section{Artigo 1: Justice at Work and Reduced Risk of Coronary Heart Disease Among} Employees- The Whitehall II Study (64)

O objetivo do estudo publicado em 2005, foi avaliar a justiça no trabalho como preditor de doença cardíaca coronariana. Foram analisados os dados 6.442 indivíduos do sexo masculino que tinham respondido às questões de justiça em pelo menos uma das duas ondas de coleta e que não tivessem histórico de doença coronariana até a segunda coleta. As mulheres foram excluídas da avaliação em função da incidência muito baixa de eventos durante o período de acompanhamento, que foi em média de 8,7 anos.

Os desfechos avaliados foram os seguintes: morte por doença arterial coronariana, primeiro infarto do miocárdio não-fatal e angina documentada.

Como cofatores foram avaliados: idade, etnia, estado marital, escolaridade, tipo de trabalho (executivo, administrativo e de apoio), colesterol sérico, índice de massa corporal, hipertensão (uso de anti-hipertensivos ou pressão arterial maior ou igual a 140/90 mmHg), uso de tabaco, consumo de álcool e atividade física. Também foram realizadas perguntas para avaliar demanda, controle e recompensa no trabalho.

Para a análise estatística foi utilizado o modelo de riscos proporcionais de Cox. Os hazard ratios (HR) e os intervalos de confiança 95\% (IC95\%) foram calculados. Trabalhadores que referenciaram altos níveis de justiça tiveram menor risco de doença arterial (HR 0,65; IC95\% 0,47-0,89) em comparação com os que referiram níveis baixo e médio de justiça, após corrigido por idade e tipo de trabalho. A associação se manteve após a inclusão de outras variáveis no modelo, como nível de colesterol, índice de massa corporal, tabagismo, hipertensão, consumo de álcool, níveis de atividade física (HR 0,71; IC95\% 0,51-0,99). Quando houve inserção de outros fatores estressores relacionados ao trabalho ao modelo estatístico (demanda controle e esforço recompensa), a associação se manteve significativa ( $\operatorname{HR} 0,69$; IC95\% 0,49-0,98). 
Outro estudo relacionado à coorte de Whitehall II, publicado em 2008, avaliou 4250 homens e 1812 mulheres livres de hipertensão arterial e doença cardíaca coronariana nas duas primeiras ondas de coleta (65). O período médio de acompanhamento foi de 9,6 anos. Foi observada a incidência de hipertensão arterial sistêmica (pressão arterial maior ou igual a 140/90 mmHg) e de doença arterial coronariana (morte por doença arterial coronariana, primeiro infarto do miocárdio nãofatal e angina documentada). Também foram acompanhados os valores de pressão de arterial sistólica, diastólica e a variação de pressão arterial ao longo do período de acompanhamento.

Após ajuste para idade, sexo, etnia e posição socioeconômica foi encontrada uma fraca correlação negativa entre justiça organizacional e média da pressão arterial diastólica (coeficiente de correlação -0,04), bem como na alteração na pressão arterial sistólica (coeficiente de correlação -0,03) e na diastólica (coeficiente de correlação 0,04). Níveis elevados de justiça organizacional demonstraram proteção para incidência de doença arterial coronariana (HR 0,87- IC95\% 0,77-0,98) quando corrigidos por idade, sexo, etnia e posição socioeconômica. Ao fazer o ajuste adicional com medidas de pressão arterial (médias de sistólica, de diastólica e variação) e hipertensão arterial sistêmica a associação se manteve (HR 0,87- IC95\% 0,77-0,98).

\section{Artigo 3: Justice at Work and Metabolic Syndrome: the Whitehall II Study (16)}

Este estudo avaliou os dados da coorte prospectiva de Whitehall II, traz as informações relativas ao seguimento de 6.123 indivíduos (4.398 homens e 1.923 mulheres) que não relataram histórico de doença cardíaca coronariana nas duas primeiras ondas de coleta. Os desfechos de interesse observados nas avaliações clínicas eram os componentes da síndrome metabólica: circunferência abdominal, triglicerídeos, colesterol HDL, pressão arterial, glicemia em jejum (ou uso de medicação antidiabética). Essas avaliações ocorreram em três ocasiões dentro de um período de aproximadamente 18 anos.

Para serem incluídos no estudo, os indivíduos não poderiam apresentar na linha de base mais que uma das seguintes alterações da avaliação clínica: colesterol total elevado ( $\geq 240 \mathrm{mg} / \mathrm{dL}$ ), diabetes (autorreferido ou em uso de medicação), obesidade (IMC $\geq 30 \mathrm{~kg} / \mathrm{m}^{2}$ ), pressão arterial elevada (sistólica $\geq 130$ ou diastólica $\geq$ $85 \mathrm{mmHg}$ ou tratamento com anti-hipertensivo). O tempo de seguimento de cada indivíduo iniciava a partir da última resposta ao questionário de justiça. $\mathrm{O}$ fim do 
seguimento dos não-casos ocorria na data da última avaliação clínica. O fim do tempo de seguimento dos indivíduos classificados como casos de síndrome metabólica (de acordo com os critérios do NCEP/ATP III e da AHA/NHLBI) era considerado o ponto médio entre a avaliação na qual foi realizado o diagnóstico e a avaliação clínica anterior. Além disso, aqueles indivíduos que apresentassem doença arterial coronariana encerravam o tempo de seguimento na data do evento, independentemente de ser diagnosticado ou não como tendo síndrome metabólica nas avaliações subsequentes. Após ajuste por idade, sexo, etnia e tipo de trabalho, foi verificado que aqueles participantes do sexo masculino que reportaram níveis altos de justiça apresentaram menor risco de desenvolver pressão arterial elevada (HR 0,86; IC95\% 0,78-0,95). Em mulheres, essa associação não foi encontrada (HR 1,02; IC95\% 0,87-1,19). Em relação ao aumento de glicemia, não foi observada associação de justiça organizacional e alteração dos níveis glicêmicos, tanto em homens (HR 1,09; IC 95\% 0,87-1,36), como em mulheres (HR 0,80; IC 95\% 0,87-1,19).

Foi realizada uma segunda análise, removendo todos os indivíduos que tinham qualquer alteração (colesterol total elevado, diabetes, obesidade ou pressão arterial elevada) na linha de base. Deste modo, foram excluídos 3305 participantes (50\% dos homens e 42,4\% das mulheres). Na nova análise, houve redução maior do risco de elevação de pressão arterial em indivíduos do sexo masculino que relataram alto nível de justiça (HR 0,79; IC 95\% 0,70-0,90).

\section{Artigo 4: Justice at work and cardiovascular mortality: a prospective cohort study (66)}

Trata-se de um estudo de coorte prospectiva, realizado na Finlândia (66), que avaliou mortalidade cardiovascular em uma amostra de 804 funcionários de diferentes tipos de trabalho e níveis hierárquicos dentro de uma indústria metalúrgica. O número de mortes foi obtido através do registro nacional finlandês, tendo sido considerados como óbitos por doenças cardiovasculares os registrados com as seguintes causas: doenças cardíacas isquêmicas, outras doenças cardíacas, doenças cerebrovasculares, outras doenças do sistema cardiovascular de acordo com a codificação da décima edição da Classificação Internacional de Doenças (CID10). Após um seguimento médio de 25,6 anos, foi verificado que os trabalhadores que foram classificados como tendo alto nível de justiça organizacional apresentaram um risco de morte $45 \%$ menor (HR 0,55- IC95\% 0,34-0,88) em comparação aos outros 
trabalhadores, que foram classificados como tendo níveis médio e baixo de justiça no modelo estatístico ajustado por idade e sexo.

Essa associação se manteve significativa (HR 0,59; IC95\% 0,36-0,96) quando houve ajuste do modelo para os seguintes fatores de risco: grupo ocupacional, tabagismo, nível de atividade física, pressão arterial sistólica e índice de massa corporal e quando outros modelos de estresse no trabalho (demanda-controle e esforço-recompensa) foram inseridos no modelo (HR 0,56- IC95\% 0,34-0,92). Quando ambos ajustes foram realizados simultaneamente, os indivíduos com alta justiça organizacional apresentaram um risco 59\% menor de ter pressão arterial elevada (HR 0,$61 ;$ IC95\% 0,36-1,00).

\section{Artigo 5: Organizational Justice and Physiological Coronary Heart Disease Risk} Factors in Japanese Employees: a Cross-Sectional Study (67)

O artigo traz os resultados da linha de base da coorte J-HOPE (Japanese study of Health, Occupationand Psychosocial factors related Equity) (67). Em uma amostra de 4.499 indivíduos (3.598 homens e 901 mulheres) em duas companhias de manufaturas, foram avaliadas as dimensões procedural e interacional da Justiça Organizacional, por meio da versão para a língua japonesa do Questionário de Justiça Organizacional de Moorman. Além do questionário autoadministrado, foram realizadas avaliações clínicas, nas quais eram mensurados pressão arterial sistólica e diastólica, peso, altura e coletados exames para avaliar lipídios séricos. As covariáveis analisadas foram: sexo, idade, nível educacional, empresa na qual o indivíduo trabalhava, ocupação, turno, doenças crônicas, estresse psicológico, uso de tabaco, uso de álcool. Após regressão logística multivariada, corrigindo para as variáveis supracitadas, não foi encontrada associação entre justiça procedural e interacional e os fatores de risco para doença cardíaca coronariana investigados entre os participantes.

Artigo 6: The moderating effect of perceived organizational support on the relationships between organizational justice and objective measures of cardiovascular health (68)

Trata-se de estudo de corte transversal realizado nos Estados Unidos (68), publicado em 2017, que avaliou 290 trabalhadores da construção civil em duas 
instituições públicas no noroeste dos Estados Unidos. Foram avaliadas as dimensões distributiva e procedural da justiça organizacional por meio da aplicação de questionários para justiça procedural (32) e justiça distributiva (40). Foi realizada avaliação clínica para verificar frequência cardíaca e pressão arterial sistólica e diastólica. As covariáveis avaliadas foram: idade, sexo, estado civil, ser responsável pelo cuidado de crianças ou idosos, número de horas trabalhadas por semana, tempo trabalhando na empresa, posição dentro do trabalho (supervisor, líder, integrante de equipe ou outros) e uso de medicamentos anti-hipertensivos. O estudo não encontrou associação entre justiça distributiva e pressão arterial sistólica ou diastólica. Em relação à dimensão procedural da justiça organizacional, encontrou-se que uma pequena correlação negativa limítrofe quando acompanhada de alta percepção de suporte organizacional. Entretanto, quando o suporte organizacional era baixo, esta associação não se manteve. 


\section{AVALIAÇÃO DO RISCO DE VIÉS}

A avaliação do risco de viés foi realizada a partir da avaliação de pontos chave dos estudos, de acordo com a ferramenta do NIH. Geralmente considera-se válida a evidência gerada pelos estudos de qualidade considerada boa. Os de qualidade razoável apresentam risco maior de vieses, mas não a ponto de invalidar os resultados encontrados. Entretanto, deve-se evitar incluir as evidências geradas por estudos considerados de qualidade ruim, em função do alto risco de viés (61). O quadro a seguir (Quadro 3) detalha os itens avaliados e a classificação final da qualidade de cada estudo selecionado para a presente revisão. 
Quadro 3- Avaliação da qualidade do estudo de acordo com os critérios do Quality Assessment Tool - NIH (traduzido pela autora).

\begin{tabular}{|c|c|c|c|c|c|c|}
\hline Critério & $\begin{array}{l}\text { Justice at Work and } \\
\text { Reduced Risk of } \\
\text { Coronary Heart } \\
\text { Disease Among } \\
\text { Employees- The } \\
\text { Whitehall II Study }\end{array}$ & $\begin{array}{l}\text { Effects on Blood } \\
\text { Pressure Do Not } \\
\text { Explain the } \\
\text { Association Between } \\
\text { Organizational Justice } \\
\text { and Coronary Heart } \\
\text { Disease in the } \\
\text { Whitehall II Study }\end{array}$ & $\begin{array}{l}\text { Justice at Work } \\
\text { and Metabolic } \\
\text { Syndrome: the } \\
\text { Whitehall II Study }\end{array}$ & $\begin{array}{l}\text { Justice at work and } \\
\text { cardiovascular } \\
\text { mortality: a } \\
\text { prospective cohort } \\
\text { study }\end{array}$ & $\begin{array}{l}\text { Organizational Justice } \\
\text { and Physiological } \\
\text { Coronary Heart Disease } \\
\text { Risk Factors in Japanese } \\
\text { Employees: a Cross- } \\
\text { Sectional Study }\end{array}$ & $\begin{array}{l}\text { The moderating effect of } \\
\text { perceived organizational support } \\
\text { on the relationships between } \\
\text { organizational justice and } \\
\text { objective measures of } \\
\text { cardiovascular health }\end{array}$ \\
\hline
\end{tabular}

1. A pergunta de pesquisa estava
descrita de maneira clara?
2. A população do estudo estava
bem definida e descrita de maneira

clara?

3. A taxa de participação de pessoas elegíveis foi de pelo menos $50 \%$ ?

4. Todos os indivíduos foram selecionados ou recrutados a partir de populações semelhantes

(incluindo a época)? Os critérios de inclusão e exclusão foram

aplicado de maneira uniforme a

todos os participantes?

5. A justificativa para o tamanho da amostra, descrição do seu poder

variância e estimativa de efeito 
6. A exposição de interesse fo medida antes da medição do

7. O período de tempo foi suficiente para que se pudesse esperar de

maneira razoável de encontrar uma

8. Para exposições que podem variar em intensidade, o estudo

avaliou diferentes níveis de

exposição em relação ao desfecho?

9. As medidas de avaliação da

exposição (variáveis

independentes) foram definidas de

maneira clara, válida, confiável e

adas de maneira

consistente entre todos os

participantes?

10. A exposição foi avaliada mais

de uma vez ao longo do tempo?

11. As medidas de avaliação do desfecho (variáveis dependentes) foram definidas de maneira clara,

válida, confiável e implementadas

de maneira consistente entre todos

$$
\text { os participantes? }
$$

12. Os avaliadores do desfecho

foram cegados em relação ao

status de exposição dos 
13. A quantidade de perdas depois da linha de base foi de $20 \%$ ou

14. As variáveis chave com

potencial de confundimento foram

medidas? Houve ajuste estatístico

para o seu impacto na relação entre

exposições e desfechos? 


\section{DISCUSSÃO}

Entre os três estudos que avaliaram doença arterial coronariana, dois deles pertencem à coorte de Whitehall. Apesar de existir uma sobreposição parcial da amostra, optou-se por manter ambos os estudos, uma vez que há diferença tanto nos grupos pesquisados e nas análises realizadas. Enquanto que no estudo de 2005 (64) a amostra era composta por 6.442 indivíduos do sexo masculino e teve um tempo médio de seguimento de 8,7 anos, o estudo de 2008 (65) tinha um total de 6.062 pessoas ( $70 \%$ sexo masculino e $30 \%$ ) após 9,6 anos de acompanhamento. Os três estudos foram considerados de qualidade boa de acordo com os critérios do NIHStudy Quality Assessment Tool. Como pontos fortes teve uma amostra bem definida, com boa taxa de resposta e baixa quantidade de perdas.

O desenho prospectivo é o mais adequado para investigações de causalidade e, sempre que possível, deve ser priorizado para avaliações desta natureza. Outro aspecto a ser levado em consideração é o tempo de seguimento compatível com o tempo de desenvolvimento da doença. Ambas as publicações apresentaram tempo médio de seguimento em torno de dez anos, o que é razoável para patologias cardiovasculares. A sua amostra, apesar de ter um tamanho considerável e ser bem caracterizada, pode ter limitações em relação à sua possibilidade de generalização. O grupo analisado, ainda que tenha variações socioeconômicas dentro da amostra, é composto por funcionários públicos, trabalhadores de escritório, que podem não representar o que acontece com outras categorias profissionais e em regimes diferentes de contratação. Além disso, a amostra era majoritariamente de cor branca (aproximadamente 90\%), vivendo em um centro urbano de um país industrializado de alta renda. Entretanto, o estudo apresenta características que fortalecem a confiança nos achados. Foi realizado ajuste para as características mais relevantes para as variáveis em questão, inclusive para os outros modelos de estresse no trabalho (Demanda-Controle e Esforço-Recompensa). Além disso, foram avaliados desfechos "duros", com medidas bastante objetivas, contribuindo para não superestimar o risco. Um aspecto a ser considerado é que, como o início da coorte foi antes do estabelecimento do modelo mais aceito de justiça organizacional e do surgimento de questionários validados, utilizou um questionário próprio, desenvolvido para estudo, 
avaliando apenas o componente interacional. Desta maneira, torna mais difícil a comparação com outros dados da literatura.

O terceiro estudo que incluiu doença arterial coronariana entre os desfechos, realizado na Finlândia (66), apresentava qualidade moderada. Foi realizado monitoramento no registro nacional finlandês de mortalidade e considerados para este fim os registros de óbito com a codificação correspondente ao capítulo de doenças do aparelho circulatório (capítulo IX) da décima edição da Classificação Internacional de Doenças (CID10). Entre os estudos identificados nesta revisão, este foi o único que incluiu doença cerebrovascular. Como o estudo não discrimina as patologias, não é possível saber se há diferença do aumento de risco entre doença coronariana e acidente vascular encefálico, por exemplo. Uma das limitações foi a avaliação da exposição apenas na linha de base e através de pergunta única (resposta à afirmação "Meu supervisor me trata de maneira justa"). Cabe ressaltar que a coleta inicial ocorreu em 1973, quando o modelo de justiça ainda estava em sua fase inicial de desenvolvimento e avaliações de item único era o mais habitual. Entre as fortalezas do estudo estão o desenho prospectivo e o longo tempo de seguimento- média de 25,6 anos- já que as doenças cardiovasculares levam alguns anos para se desenvolver a partir do início da exposição. Foram realizados ajustes para os principais fatores de risco, como idade, sexo, tabagismo, atividade física, pressão arterial sistólica e colesterol. A amostra, composta por 804 indivíduos de uma indústria metalúrgica, apresentava diversidade de tipos de trabalho (entre especializados e não especializados), incluindo os trabalhadores do chamado "blue collar".

A avaliação de associação de justiça organizacional com alteração da pressão arterial foi descrita em quatro estudos, que apresentam alguns resultados conflitantes. Dados da coorte de Whitehall, que avaliaram os aspectos interacionais da Justiça Organizacional, inicialmente encontraram uma fraca correlação negativa entre a percepção de alta justiça e a média da pressão arterial diastólica. Também houve fraca correlação negativa com a alteração da pressão sistólica e da diastólica após um período médio de acompanhamento de 9,6 anos. Em uma publicação subsequente, foi verificado que os homens que relatavam altos níveis de justiça tiveram um risco 14\% menor de desenvolver pressão arterial elevada, após um seguimento médio de 18 anos. Os outros dois estudos que estudaram pressão arterial eram de desenho transversal. No estudo conduzido no Japão (67) foram avaliadas as dimensões procedural e interacional, enquanto nos dos Estados Unidos (68), foram 
estudadas as dimensões distributivas e procedural da Justiça Organizacional. Em ambos os casos, não foi encontrada associação entre hipertensão arterial e justiça. Existe uma dificuldade em comparar esses dados, uma vez que os estudos têm desenhos diferentes, não avaliaram as mesmas dimensões da justiça, nem tampouco utilizaram os mesmos instrumentos. É razoável supor que a associação seja de pouca magnitude e apareça apenas após muitos anos de exposição.

Somente um estudo avaliou a relação entre justiça organizacional e diabetes mellitus. Este foi considerado como tendo boa qualidade. Gimeno et. al não encontraram associação entre justiça interacional e alteração nos níveis de glicêmicos tanto em homens, como em mulheres, após 18 anos de acompanhamento. 


\section{CONSIDERAÇÕES FINAIS}

Esta pesquisa se propôs a revisar sistematicamente o estresse no trabalho pelo modelo de Justiça Organizacional e desfechos negativos em saúde (cardiovascular e metabólicos). Foi observado que ainda são poucos os estudos disponíveis com este enfoque. Recentemente, foi publicada uma análise bibliométrica que buscou avaliar as tendências de pesquisas dentro do campo da Justiça Organizacional (69). Identificou-se um aumento progressivo do interesse, com mais da metade de todas as publicações tendo ocorrido na última década. A maior concentração ocorre nas áreas de ciências sociais, administração, psicologia e humanidades, estando a medicina em quinto lugar no número de publicações, apesar de se destacar em quantidade de citações. Cerca de 95\% das publicações são em língua inglesa, havendo destaque da participação de países europeus. Dentro da área da saúde, são frequentes os estudos avaliando ansiedade, depressão, alterações de sono, burnout e absenteísmo. Sendo assim, o resultado obtido na busca de estudos para a presente revisão parece estar de acordo com o que há de disponível na literatura científica.

Com relação aos desfechos selecionados, os resultados estão com o padrão semelhante ao que foi encontrado em estudos prévios $(22,54)$. Os dados mais consistentes são os relativos a doenças cardiovasculares, em especial a doença arterial coronariana. Houve dificuldade de comparar os resultados dos estudos já que os instrumentos utilizados e as dimensões da justiça organizacional investigada variaram.

Os quatro estudos de coorte avaliados na presente revisão mostraram que houve associação entre justiça organizacional alta e menor risco de doenças cardiovasculares e de eventos fatais por essas doenças. Por outro lado, não foram encontradas associações entre justiça organizacional e níveis de glicemia.

Futuros estudos devem incluir investigação de Justiça Organizacional e desfechos em saúde em de países de baixa e média rendas, incluindo trabalhadores de diversas áreas, não apenas servidores públicos, da indústria e da construção civil, o estudo da variável raça/cor. Além disso, a investigação das três dimensões da justiça organizacional pode fornecer informações relevantes para elaboração de ações e de políticas nas instituições voltadas para aumentar os níveis de justiça organizacional. 


\section{REFERÊNCIAS}

1. Rom O, Reznick AZ. The Stress Reaction: A Historical Perspective. In 2015. p. $1-4$.

2. Levi L, Bartley M, Marmot M, Karasek R, Theorell T, Siegrist J, et al. Stressors at the workplace: theoretical models. Occup Med. 2000;

3. McEwen BS. Stress, Adaptation, and Disease: Allostasis and Allostatic Load. Ann N Y Acad Sci [Internet]. maio de 1998;840(1):33-44.

4. Sterling,Peter Eyer J. Allostasis: a new paradigm to explain arousal pathology. In: Fisher, S. Reason J, organizador. Handbook of life stress, cognition and health. John Wiley \& Sons; 1988. p. 629-49.

5. Rozanski A, Blumenthal JA, Kaplan J. Impact of Psychological Factors on the Pathogenesis of Cardiovascular Disease and Implications for Therapy. Circulation [Internet]. 27 de abril de 1999;99(16):2192-217.

6. Peter R, Siegrist J. Psychosocial work environment and the risk of coronary heart disease. Int Arch Occup Environ Health [Internet]. 2000.

7. Jahoda M. Employment and Unemployment [Internet]. Cambridge: Cambridge University Press; 1982.

8. Morris JK, Cook DG, Shaper AG. Loss of employment and mortality. BMJ [Internet]. 30 de abril de 1994;308(6937):1135-9.

9. Stefansson C-G. Long-term unemployment and mortality in Sweden, 19801986. Soc Sci Med [Internet]. janeiro de 1991;32(4):419-23.

10. Martikainen PT. Unemployment and mortality among Finnish men, 1981-5. BMJ [Internet]. 1 de setembro de 1990;301(6749):407-11.

11. International Labour Organization (ILO). Workplace Stress: A collective challenge [Internet]. International Labour Organization, organizador. Geneva: International Training Centre of the International Labour Organization; 2016. 1$63 \mathrm{p}$.

12. Karasek R, Theorell T. Healthy Work: Stres, Productivity, and the Reconstruction of Working Life. Journal of Industrial Relations. 1990.

13. Siegrist J. Adverse health effects of high-effort/low-reward conditions. J Occup Health Psychol [Internet]. 1996;1(1):27-41.

14. Elovainio M, Ferrie JE, Gimeno D, De Vogli R, Shipley M, Brunner EJ, et al. Organizational justice and sleeping problems: The whitehall II study. Psychosom Med [Internet]. 2009. 
15. Kouvonen A, Kivimäki M, Elovainio M, Väänänen A, De Vogli R, Heponiemi T, et al. Low organisational justice and heavy drinking: a prospective cohort study. Occup Environ Med [Internet]. 1 de janeiro de 2008;65(1):44 LP - 50.

16. Gimeno D, Tabak AG, Ferrie JE, Shipley MJ, De Vogli R, Elovainio M, et al. Justice at work and metabolic syndrome: the Whitehall II study. Occup Environ Med [Internet]. 1 de abril de 2010;67(4):256-62.

17. Kivimäki M, Vahtera J, Elovainio M, Virtanen M, Siegrist J. Effort-reward imbalance, procedural injustice and relational injustice as psychosocial predictors of health: Complementary or redundant models? Occup Environ Med. 2007;64(10):659-65.

18. Kivimäki M, Elovainio M, Vahtera J, Virtanen M, Stansfeld SA. Association between organizational inequity and incidence of psychiatric disorders in female employees. Psychol Med. 2003;

19. World Health Organization (WHO). Stress at the workplace [Internet]. Geneva. 2014. Available at: http://www.who.int/occupational_health/topics/stressatwp/en/

20. Gimeno D, Tabák ÁG, Ferrie JE, Shipley MJ, De Vogli R, Elovainio M, et al. Justice at work and metabolic syndrome: The Whitehall II study. Occup Environ Med. 2010;

21. Tsai S-S, Lai C-H, Shih T-S, Lin M-H, Liou S-H. High job strain is associated with inflammatory markers of disease in young long-haul bus drivers. J Occup Health Psychol [Internet]. 2014.

22. Sara JD, Prasad M, Eleid MF, Zhang M, Jay Widmer R, Lerman A. Association between work-related stress and coronary heart disease: A review of prospective studies through the job strain, effort-reward balance, and organizational justice models. J Am Heart Assoc. 2018;7(9):1-16.

23. Nieuwenhuijsen K, Bruinvels D, Frings-Dresen M. Psychosocial work environment and stress-related disorders, a systematic review. Occup Med (Lond). 2010;60(4):277-86.

24. Hjarsbech PU, Christensen KB, Bjorner JB, Madsen IEH, Thorsen S V., Carneiro IG, et al. A multi-wave study of organizational justice at work and long-term sickness absence among employees with depressive symptoms. Scand J Work Environ Heal. 2014;40(2):176-85.

25. Leineweber C, Bernhard-Oettel C, Peristera P, Eib C, Nyberg A, Westerlund H. Interactional justice at work is related to sickness absence: a study using repeated measures in the Swedish working population. BMC Public Health. 2017;17(1):912.

26. Spanier K, 1 2, Radoschewski F, Gutenbrunner C, Bethge M, 1 2. Direct and indirect effects of organizational justice on work ability. Occup Med. 2014; 
27. Wang J, Schmitz N. Does job strain interact with psychosocial factors outside of the workplace in relation to the risk of major depression? The Canadian National Population Health Survey. Soc Psychiatry Psychiatr Epidemiol [Internet]. 21 de julho de 2011;46(7):577-84.

28. Assunção AÁ, Machado CJ, Prais HAC, de Araújo TM. Depression among physicians working in public healthcare in Belo Horizonte, Brazil. Soc Psychiatry Psychiatr Epidemiol [Internet]. 7 de agosto de 2014;49(8):1219-29.

29. Da Silva ATC, De Souza Lopes C, Susser E, Menezes PR. Work-related depression in primary care teams in Brazil. Am J Public Health. 2016;106(11):1990-7.

30. Siegrist J. Adverse health effects of high-effort/low-reward conditions. Journal of occupational health psychology. 1996.

31. Greenberg J. Organizational Justice: Yesterday, Today, and Tomorrow. J Manage. 1990;16(2):399-432.

32. Moorman R. Relationship between organizational justice and organizational citizenship behaviors: Do fairness perceptions influence employee citizenship. J Appl Psychol. 1991;76(6):845-55.

33. Miller DT. Disrespect and the Experience of Injustice. Annu Rev Psychol. 2001;

34. Greenberg J, Colquitt J. What Is Organizational Justice? A Historical Overview. In: Greenberg J, Colquitt JA, organizadores. Handbook of Organizational Justice. Mahwah, NJ, US: Lawrence Erlbaum Associates Publishers; 2005. p. 3-56.

35. Greenberg J. Organizational justice: The dynamics of fairness in the workplace. APA Handb Ind Organ Psychol [Internet]. 2011;3:271-327.

36. Leventhal GS. What Should Be Done with Equity Theory? In: Social Exchange [Internet]. Boston, MA: Springer US; 1980. p. 27-55.

37. Bies RJ, Shapiro DL. Interactional fairness judgments: The influence of causal accounts. Soc Justice Res [Internet]. junho de 1987;1(2):199-218.

38. Moliner C, Cropanzano R, Martínez-Tur V. Organizational Justice: International perspectives and conceptual advances. $1^{\text {a }}$. Moliner C, Cropanzano R, Martínez-Tur V, organizadores. New York, NY: Routledge; 2017.

39. Colquitt J, Shaw JC. How should Organizational Justice be measured? In: Greenberg J, Colquitt JA, organizadores. Handbook of Organizational Justice. Mahwah, NJ, US: Lawrence Erlbaum Associates Publishers; 2005. p. 113-52.

40. Price J, Mueller C. Distributive Justice. In: Handbook of Organizational Measurement [Internet]. Marshfield, MA: Pitman; 1986. p. 122-7. 
41. Elovainio M, Kivimäki M, Vahtera J. Organizational Justice: Evidence of a New Psychosocial Predictor of Health. Am J Public Health [Internet]. janeiro de 2002;92(1):105-8.

42. Mendonça H, Pereira C, Tamayo Á, Paz M das GT da. Validação Fatorial de uma Escala de Percepção de Justiça Organizacional. Vol. 30, Estudos Goiânia. 2003. p. 111-30.

43. Colquitt JA. On the dimensionality of organizational justice: A construct validation of a measure. J Appl Psychol [Internet]. junho de 2001;86(3): 386400.

44. Schmitt M, Dörfel M. Procedural injustice at work, justice sensitivity, job satisfaction and psychosomatic well-being. Eur J Soc Psychol [Internet]. junho de 1999;29(4):443-53.

45. Tepper BJ. Health Consequences of Organizational Injustice: Tests of Main and Interactive Effects. Organ Behav Hum Decis Process [Internet]. novembro de 2001;86(2):197-215.

46. Gürbüz S, Mert SI. Validity and reliability tests of organizational justice scale: An empirical study in a public organization. Today's Rev Public Adm [Internet]. 2017;3(3):137-62.

47. World Health Organization (WHO). Cardiovascular diseases (CVDs) [Internet]. 2017. Available at: https://www.who.int/en/news-room/factsheets/detail/cardiovascular-diseases-(cvds)

48. Roth GA, Abate D, Abate KH, Abay SM, Abbafati C, Abbasi N, et al. Global, regional, and national age-sex-specific mortality for 282 causes of death in 195 countries and territories, 1980-2017: a systematic analysis for the Global Burden of Disease Study 2017. Lancet [Internet]. novembro de 2018;392(10159):1736-88.

49. Beaglehole $R$, Magnus $P$. The search for new risk factors for coronary heart disease: occupational therapy for epidemiologists? Int J Epidemiol [Internet]. dezembro de 2002;31(6):1117-22.

50. Hall JE. Guyton \& Hall- Tratado de Fisiologia Médica. 13a . John E. Hall, organizador. Rio de Janeiro: Guanabara Koogan; 2021.

51. Marmot MG, Wilkinson RG. Social Determinants of Health. 2a ${ }^{a}$ Marmot MG, Wilkinson RG, organizadores. New York, NY: Oxford University Press; 2006.

52. Weber CS, Thayer JF, Rudat M, Wirtz PH, Zimmermann-Viehoff F, Thomas A, et al. Low vagal tone is associated with impaired post stress recovery of cardiovascular, endocrine, and immune markers. Eur J Appl Physiol [Internet]. 7 de maio de 2010;109(2):201-11. 
53. Jarczok MN, Jarczok M, Mauss D, Koenig J, Li J, Herr RM, et al. Autonomic nervous system activity and workplace stressors-A systematic review. Neurosci Biobehav Rev [Internet]. setembro de 2013;37(8):1810-23.

54. Kivimäki M, Virtanen M, Elovainio $M$, Kouvonen A, Väänänen A, Vahtera J. Work stress in the etiology of coronary heart disease-a meta-analysis. Scand J Work Environ Health [Internet]. dezembro de 2006;32(6):431-42.

55. Kasl $\mathrm{S} V$. The influence of the work environment on cardiovascular health: $\mathrm{A}$ historical, conceptual, and methodological perspective. J Occup Health Psychol [Internet]. 1996;1(1):42-56.

56. Van der Klink JJL, Blonk RWB, Schene AH, Van Dijk FJH. The benefits of interventions for work-related stress. Am J Public Health. 2001;

57. Linden W. Psychosocial Interventions for Patients With Coronary Artery Disease. Arch Intern Med [Internet]. 8 de abril de 1996;156(7):745.

58. LaMontagne AD, Keegel T, Louie AM, Ostry A, Landsbergis PA. A systematic review of the job-stress intervention evaluation literature, 1990-2005. International Journal of Occupational and Environmental Health. 2007.

59. Center for Reviews and Dissemination. Systematic Reviews - CRD's guidelines for undertaking reviews in healthcare. Centre for Reviews and Dissemination, University of York. 2009.

60. Moher D, Shamseer L, Clarke M, Ghersi D, Liberati A, Petticrew M, et al. Preferred reporting items for systematic review and meta-analysis protocols (PRISMA-P) 2015 statement. Syst Rev [Internet]. 1 de dezembro de 2015;4(1):1.

61. National Institute of Health (NIH). National Institute of Health (NIH) quality assessment tools. [Internet]. Available at: https://www.nhlbi.nih.gov/healthtopics/study-quality-assessment-tools

62. Institute of Epidemiology \& Health Care- University College London. Whitehall II [Internet]. Available at: https://www.ucl.ac.uk/epidemiology-healthcare/research/epidemiology-and-public-health/research/whitehall-ii

63. Marmot M, Brunner E. Cohort Profile: The Whitehall II study. Int J Epidemiol [Internet]. 1 de abril de 2005;34(2):251-6.

64. Kivimäki M, Ferrie JE, Brunner E, Head J, Shipley MJ, Vahtera J, et al. Justice at Work and Reduced Risk of Coronary Heart Disease Among Employees. Arch Intern Med [Internet]. 24 de outubro de 2005;165(19):2245.

65. Kivimäki M, Ferrie JE, Shipley M, Gimeno D, Elovainio M, de Vogli R, et al. Effects on Blood Pressure Do Not Explain the Association Between Organizational Justice and Coronary Heart Disease in the Whitehall II Study. Psychosom Med [Internet]. janeiro de 2008;70(1):1-6. 
66. Elovainio $M$, Leino-Arjas $P$, Vahtera J, Kivimäki M. Justice at work and cardiovascular mortality: a prospective cohort study. J Psychosom Res [Internet]. agosto de 2006;61(2):271-4.

67. Inoue A, Kawakami N, Eguchi H, Miyaki K, Tsutsumi A. Organizational Justice and Physiological Coronary Heart Disease Risk Factors in Japanese Employees: a Cross-Sectional Study. Int J Behav Med [Internet]. 20 de dezembro de 2015;22(6):775-85.

68. Rineer JR, Truxillo DM, Bodner TE, Hammer LB, Kraner MA. The moderating effect of perceived organizational support on the relationships between organizational justice and objective measures of cardiovascular health. Eur $\mathrm{J}$ Work Organ Psychol [Internet]. 4 de maio de 2017;26(3):399-410.

69. Sheeraz MI, Ungku Ahmad UN, Ishaq MI, Sarfraz M, Md. Nor K. The Research on Organizational Justice in Scopus Indexed Journals: A Bibliometric Analysis of Seven Decades. Front Psychol [Internet]. 10 de junho de 2021;12. 


\section{ANEXO}

\section{Aprovação do Comitê de Ética}

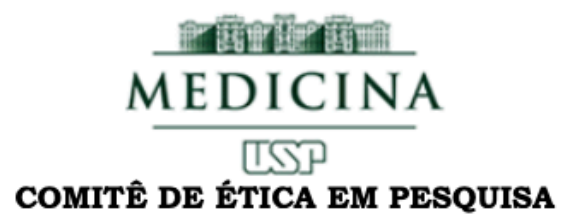

\section{APROVAÇÃO}

O Comitê de Ética em Pesquisa da Faculdade de Medicina da Universidade de São Paulo, em sessão de 22/01/2020, APROVOU o Protocolo de Pesquisa no 004/20 intitulado: Justiça Organizacional e desfechos negativos de saúde: uma revisão sistemática. Apresentado pelo Departamento Medicina Preventiva.

Cabe ao pesquisador elaborar e apresentar ao CEPFMUSP, os relatórios parciais e final sobre a pesquisa (Resolução do Conselho Nacional de Saúde no 466/12, inciso IX.2, letra "c").

Pesquisador (a) Responsável: Prof. Dr. Paulo Rossi Menezes

Pesquisador (a) Executante: Angela Scalabrin

CEP-FMUSP, 22 de Janeiro de 2020.

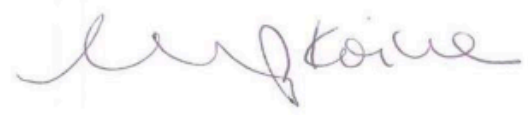

Profa. Dra. Maria Aparecida Azevedo Koike Folgueira Coordenador Comitê de Ética em Pesquisa 


\section{APÊNDICES}

\section{APÊNDICE A- Estratégia de Busca- PubMed}

\section{\#Combinação 1- Justiça Organizacional e Doença Arterial Coronariana}

$(((()((()(()((()$ "Organizational injustice") OR ("Organizational justice")) OR ("Organisational injustice")) OR ("Organisational justice")) OR ("Distributive injustice")) OR ("Distributive justice")) OR ("Procedural injustice")) OR ("Procedural justice")) OR ("Interactional injustice")) OR ("Interactional justice")) OR ("Relational injustice")) OR ("Relational justice")) OR ("Job stress")) OR ("Work stress")) OR ("Work distress")) OR ("Occupational stress")) OR ("Psychosocial work conditions")) AND (((((("Ischemic Heart Disease") OR ("Acute coronary syndrome")) OR ("Coronary artery disease")) OR ("Coronary disease")) OR ("Myocardial infarction")) OR (Angina)) OR ("Ischemic Heart Disease"[All Fields] OR "Acute coronary syndrome"[All Fields] OR "Coronary artery disease"[All Fields] OR "Coronary disease"[All Fields] OR "Myocardial infarction"[All Fields] OR "angina pectoris"[MeSH Terms]))

\section{\#Combinação 2- Justiça Organizacional e Doença Cerebrovascular}

$((()((()((()((()$ Organizational injustice") $\quad$ OR $\quad$ ("Organizational justice")) OR ("Organisational injustice")) OR ("Organisational justice")) OR ("Distributive injustice")) OR ("Distributive justice")) OR ("Procedural injustice")) OR ("Procedural justice")) OR ("Interactional injustice")) OR ("Interactional justice")) OR ("Relational injustice")) OR ("Relational justice")) OR ("Job stress")) OR ("Work stress")) OR ("Work distress")) OR ("Occupational stress")) OR ("Psychosocial work conditions")) AND (((((("Cerebrovascular disease") OR ("Cerebrovascular disorders")) OR ("Acute ischemic attack")) OR (Stroke)) OR ("Brain infarction")) OR ("Cerebrovascular disease" OR "Cerebrovascular disorders" OR "Acute ischemic attack" OR Stroke OR "Brain infarction"[MeSH Terms]))

\section{\#Combinação 3- Justiça Organizacional e Hipertensão Arterial Sistêmica}

((()((()((()(((("Organizational injustice") $\quad$ OR $\quad$ ("Organizational justice")) OR ("Organisational injustice")) OR ("Organisational justice")) OR ("Distributive injustice")) OR ("Distributive justice")) OR ("Procedural injustice")) OR ("Procedural justice")) OR 
("Interactional injustice")) OR ("Interactional justice")) OR ("Relational injustice")) OR ("Relational justice")) OR ("Job stress")) OR ("Work stress")) OR ("Work distress")) OR ("Occupational stress")) OR ("Psychosocial work conditions")) AND (((Hypertension) OR ("High blood pressure")) OR (Hypertension OR "High blood pressure"[MeSH Terms]))

\section{\#Combinação 4- Justiça Organizacional e Diabetes Mellitus}

$((()((()((()((()$ Organizational injustice") $\quad$ OR $\quad$ ("Organizational justice")) $\quad$ OR ("Organisational injustice")) OR ("Organisational justice")) OR ("Distributive injustice")) OR ("Distributive justice")) OR ("Procedural injustice")) OR ("Procedural justice")) OR ("Interactional injustice")) OR ("Interactional justice")) OR ("Relational injustice")) OR ("Relational justice")) OR ("Job stress")) OR ("Work stress")) OR ("Work distress")) OR ("Occupational stress")) OR ("Psychosocial work conditions")) AND ((("Type 2 diabetes") OR (Diabetes)) OR ("Type 2 diabetes" OR Diabetes[MeSH Terms]))

\section{\#Combinação 5- Justiça Organizacional e Doença Cardiovascular}

((()((()((()((((")rganizational injustice") $\quad$ OR $\quad$ ("Organizational justice")) $\quad$ OR ("Organisational injustice")) OR ("Organisational justice")) OR ("Distributive injustice")) OR ("Distributive justice")) OR ("Procedural injustice")) OR ("Procedural justice")) OR ("Interactional injustice")) OR ("Interactional justice")) OR ("Relational injustice")) OR ("Relational justice")) OR ("Job stress")) OR ("Work stress")) OR ("Work distress")) OR ("Occupational stress")) OR ("Psychosocial work conditions")) AND ((("Cardiovascular diseases")) OR ("Cardiovascular diseases"[MeSH Terms]))

\section{APÊNDICE B- Estratégia de busca- EMBASE}

\section{\#Combinação 1- Justiça Organizacional e Doença Arterial Coronariana}

('organizational injustice' OR 'organizational justice' OR 'organisational injustice' OR 'organisational justice' OR 'distributive injustice' OR 'distributive justice'/exp OR 'distributive justice' OR 'procedural injustice' OR 'procedural justice'/exp OR 'procedural justice' OR 'interactional injustice' OR 'interactional justice' OR 'relational injustice' OR 'relational justice' OR 'job stress'/exp OR 'job stress' OR 'work stress'/exp 
OR 'work stress' OR 'work distress' OR 'occupational stress'/exp OR 'occupational stress' OR 'psychosocial work conditions' OR 'organizational justice'/exp) AND ('ischemic heart disease' OR 'acute coronary syndrome' OR 'coronary artery disease' OR 'coronary disease'/exp OR 'coronary disease' OR 'myocardial infarction'/exp OR 'myocardial infarction' OR 'angina'/exp OR angina OR 'ischemic heart disease'/exp OR 'acute coronary syndrome'/exp OR 'coronary artery disease'/exp OR 'heart infarction'/exp OR 'angina pectoris'/exp)

\section{\#Combinação 2- Justiça Organizacional e Doença Cerebrovascular}

('organizational injustice' OR 'organizational justice' OR 'organisational injustice' OR 'organisational justice' OR 'distributive injustice' OR 'distributive justice'/exp OR 'distributive justice' OR 'procedural injustice' OR 'procedural justice'/exp OR 'procedural justice' OR 'interactional injustice' OR 'interactional justice' OR 'relational injustice' OR 'relational justice' OR 'job stress'/exp OR 'job stress' OR 'work stress'/exp OR 'work stress' OR 'work distress' OR 'occupational stress'/exp OR 'occupational stress' OR 'psychosocial work conditions' OR 'organizational justice'/exp) AND ('cerebrovascular disease' OR 'cerebrovascular disorders'/exp OR 'cerebrovascular disorders' OR 'acute ischemic attack' OR 'stroke'/exp OR stroke OR 'brain infarction' OR 'cerebrovascular disease'/exp OR 'cerebrovascular accident'/exp OR 'brain infarction'/exp)

\section{\#Combinação 3- Justiça Organizacional e Hipertensão Arterial Sistêmica} ('organizational injustice' OR 'organizational justice' OR 'organisational injustice' OR 'organisational justice' OR 'distributive injustice' OR 'distributive justice'/exp OR 'distributive justice' OR 'procedural injustice' OR 'procedural justice'/exp OR 'procedural justice' OR 'interactional injustice' OR 'interactional justice' OR 'relational injustice' OR 'relational justice' OR 'job stress'/exp OR 'job stress' OR 'work stress'/exp OR 'work stress' OR 'work distress' OR 'occupational stress'/exp OR 'occupational stress' OR 'psychosocial work conditions' OR 'organizational justice'/exp) AND (hypertension OR 'high blood pressure'/exp OR 'high blood pressure' OR 'hypertension'/exp

\section{\#Combinação 4- Justiça Organizacional e Diabetes Mellitus}


('organizational injustice' OR 'organizational justice' OR 'organisational injustice' OR 'organisational justice' OR 'distributive injustice' OR 'distributive justice'/exp OR 'distributive justice' OR 'procedural injustice' OR 'procedural justice'/exp OR 'procedural justice' OR 'interactional injustice' OR 'interactional justice' OR 'relational injustice' OR 'relational justice' OR 'job stress'/exp OR 'job stress' OR 'work stress'/exp OR 'work stress' OR 'work distress' OR 'occupational stress'/exp OR 'occupational stress' OR 'psychosocial work conditions' OR 'organizational justice'/exp) AND ('type 2 diabetes'/exp OR 'type 2 diabetes' OR 'diabetes'/exp OR diabetes OR 'non insulin dependent diabetes mellitus'/exp)

\#Combinação 5- Justiça Organizacional e Doença Cardiovascular ('organizational injustice' OR 'organizational justice' OR 'organisational injustice' OR 'organisational justice' OR 'distributive injustice' OR 'distributive justice'/exp OR 'distributive justice' OR 'procedural injustice' OR 'procedural justice'/exp OR 'procedural justice' OR 'interactional injustice' OR 'interactional justice' OR 'relational injustice' OR 'relational justice' OR 'job stress'/exp OR 'job stress' OR 'work stress'/exp OR 'work stress' OR 'work distress' OR 'occupational stress'/exp OR 'occupational stress' OR 'psychosocial work conditions' OR

'organizational justice'/exp) AND ('cardiovascular diseases'/exp OR 'cardiovascular diseases' OR 'cardiovascular diseases etiology'/exp)

\section{APÊNDICE C- Estratégia de Busca- LILACS}

\#Combinação 1- Justiça Organizacional e Doença Arterial Coronariana (("Justiça organizacional" OR "Justiça distributiva" OR "Justiça processual" OR "Justiça relacional" OR "Estresse no trabalho" OR "Estresse ocupacional" OR "Organizational justice" OR "Distributive justice" OR "Procedural justice" OR "Relational justice" OR "Job stress" OR "Work stress" OR "Work distress" OR "Occupational stress" OR "Justicia organizacional" OR "Justicia distributiva" OR "Justicia procesal" OR "Estrés laboral" OR "Estrés ocupacional" OR "Estrés en el trabajo" OR "Justicia organizacional" OR "Justicia distributiva" OR "Justicia procesal" OR "Estrés laboral" OR "Estrés ocupacional" OR "Estrés en el trabajo" ) OR (mh:(Estresse Ocupacional))) AND (("Doença cardíaca isquêmica" OR "Síndrome coronariana aguda" OR "Doença 
da arterial coronariana" OR "Doença da Artéria Coronariana" OR "Doença coronariana" OR "Infarto do miocárdio" OR "Infarto agudo do miocárdio" OR "Angina pectoris" OR "Ischemic Heart Disease" OR "Acute coronary syndrome" OR "Coronary artery disease" OR "Coronary disease" OR "Myocardial infarction" OR "Acute Myocardial infarction" OR "Síndrome coronario agudo" OR "Enfermedad de la Arteria Coronaria" OR "Enfermedad Coronaria" OR "Infarto del Miocardio" OR "Infarto Agudo del Miocardio" OR "angina de pecho" ) OR (mh:(Doença da Artéria Coronariana)) OR (mh:(Doença das Coronárias)) OR (mh:(Infarto do Miocárdio)) OR (mh:(Isquemia Miocárdica)) OR (mh:(Síndrome Coronariana Aguda)) OR (mh:(Angina Pectoris)))

\section{\#Combinação 2- Justiça Organizacional e Doença Cerebrovascular}

(("Justiça organizacional" OR "Justiça distributiva" OR "Justiça processual" OR "Justiça relacional" OR "Estresse no trabalho" OR "Estresse ocupacional" OR "Organizational justice" OR "Distributive justice" OR "Procedural justice" OR "Relational justice" OR "Job stress" OR "Work stress" OR "Work distress" OR "Occupational stress" OR "Justicia organizacional" OR "Justicia distributiva" OR "Justicia procesal" OR "Estrés laboral" OR "Estrés ocupacional" OR "Estrés en el trabajo" OR "Justicia organizacional" OR "Justicia distributiva" OR "Justicia procesal" OR "Estrés laboral" OR "Estrés ocupacional" OR "Estrés en el trabajo" ) OR (mh:(Estresse Ocupacional))) AND (("Doença cerebrovascular" OR "Transtornos Cerebrovasculares" OR "Acidente Vascular Cerebral" OR "Acidente Vascular Encefálico" OR "Infarto Cerebral" OR "Cerebrovascular disease" OR "Cerebrovascular disorders" OR "Acute ischemic attack" OR Stroke OR "Brain infarction" OR "Trastornos Cerebrovasculares" OR "Accidente Cerebrovascular" OR "Accidente Vascular Cerebral" OR "Accidente Vascular Encefálico" ) OR (mh:(Acidente Vascular Cerebral)) OR (mh:(Infarto Cerebral)) OR (mh:(Infarto Encefálico)) OR (mh:(Transtornos Cerebrovasculares)))

\section{\#Combinação 3- Justiça Organizacional e Hipertensão Arterial Sistêmica} (("Justiça organizacional" OR "Justiça distributiva" OR "Justiça processual" OR "Justiça relacional" OR "Estresse no trabalho" OR "Estresse ocupacional" OR "Organizational justice" OR "Distributive justice" OR "Procedural justice" OR "Relational justice" OR "Job stress" OR "Work stress" OR "Work distress" OR "Occupational stress" OR "Justicia organizacional" OR "Justicia distributiva" OR 
"Justicia procesal" OR "Estrés laboral" OR "Estrés ocupacional" OR "Estrés en el trabajo" OR "Justicia organizacional" OR "Justicia distributiva" OR "Justicia procesal" OR "Estrés laboral" OR "Estrés ocupacional" OR "Estrés en el trabajo" ) OR (mh:(Estresse Ocupacional))) AND (("Hipertensão Arterial Sistêmica" OR "Hipertensão Arterial" OR Hypertension OR "High blood pressure" OR Hipertensión) OR (mh:(Hipertensão)))

\section{\#Combinação 4- Justiça Organizacional e Diabetes Mellitus}

(("Justiça organizacional" OR "Justiça distributiva" OR "Justiça processual" OR "Justiça relacional" OR "Estresse no trabalho" OR "Estresse ocupacional" OR "Organizational justice" OR "Distributive justice" OR "Procedural justice" OR "Relational justice" OR "Job stress" OR "Work stress" OR "Work distress" OR "Occupational stress" OR "Justicia organizacional" OR "Justicia distributiva" OR "Justicia procesal" OR "Estrés laboral" OR "Estrés ocupacional" OR "Estrés en el trabajo" OR "Justicia organizacional" OR "Justicia distributiva" OR "Justicia procesal" OR "Estrés laboral" OR "Estrés ocupacional" OR "Estrés en el trabajo" ) OR (mh:(Estresse Ocupacional))) AND (("Diabetes Mellitus" OR "Diabetes Mellitus tipo 2" OR "Diabetes tipo 2" OR "Type 2 diabetes" OR Diabetes) OR (mh:(Diabetes Mellitus)) OR (mh:(Diabetes Mellitus Tipo 2)))

\section{\#Combinação 5- Justiça Organizacional e Doença Cardiovascular}

(("Justiça organizacional" OR "Justiça distributiva" OR "Justiça processual" OR "Justiça relacional" OR "Estresse no trabalho" OR "Estresse ocupacional" OR "Organizational justice" OR "Distributive justice" OR "Procedural justice" OR "Relational justice" OR "Job stress" OR "Work stress" OR "Work distress" OR "Occupational stress" OR "Justicia organizacional" OR "Justicia distributiva" OR "Justicia procesal" OR "Estrés laboral" OR "Estrés ocupacional" OR "Estrés en el trabajo" OR "Justicia organizacional" OR "Justicia distributiva" OR "Justicia procesal" OR "Estrés laboral" OR "Estrés ocupacional" OR "Estrés en el trabajo" ) OR (mh:(Estresse Ocupacional))) AND (("Doenças Cardiovasculares" OR "Cardiovascular diseases" OR "Enfermedades Cardiovasculares") OR (mh:(Doenças Cardiovasculares))) 